\title{
A new factorization technique of the matrix mask of univariate refinable functions
}

\section{Gerlind Plonka}

\author{
Fachbereich Mathematik \\ Universität Duisburg \\ D-47048 Duisburg, Germany \\ plonka@math.uni-duisburg.de
}

\section{Amos Ron}

\author{
Computer Science Department \\ University of Wisconsin-Madison \\ 1210 West Dayton Street \\ Madison, Wisconsin 53706, USA \\ amos@cs.wisc.edu
}

\begin{abstract}
A univariate compactly supported refinable function $\phi$ can always be factored into $B_{k} * f$, with $B_{k}$ the B-spline of order $k, f$ a compactly supported distribution, and $k$ the approximation orders provided by the underlying shift-invariant space $S(\phi)$.

Factorizations of univariate refinable vectors $\Phi$ were also studied and utilized in the literature. One of the by-products of this article is a rigorous analysis of that factorization notion, including, possibly, the first precise definition of that process.

The main goal of this article is the introduction of a special factorization algorithm of refinable vectors that generalizes the scalar case as closely (and unexpectedly) as possible: the original vector $\Phi$ is shown to be 'almost' in the form $B_{k} * F$, with $F$ still compactly supported and refinable, and $k$ the approximation order of $S(\Phi)$ : 'almost' in the sense that $\Phi$ and $B_{k} * F$ differ at most in one entry. The algorithm guarantees $F$ to retain the possible favorable properties of $\Phi$, such as the stability of the shifts of $\Phi$ and/or the polynomiality of the mask symbol. At the same time, the theory and the algorithm are derived under relatively mild conditions and, in particular, apply to $\Phi$ whose shifts are not stable, as well as to refinable vectors which are not compactly supported.

The usefulness of this specific factorization for the study of the smoothness of FSI wavelets (known also as 'multiwavelets' and 'multiple wavelets') is explained.

The analysis invokes in an essential way the theory of finitely generated shift-invariant (FSI) spaces, and, in particular, the tool of superfunction theory.
\end{abstract}

AMS (MOS) Subject Classifications: Primary 42C15, 42A85, Secondary 41A25, 46E35

Key Words: FSI spaces, refinable vectors, factorization of masks, approximation order, multiwavelets.

This work was supported by the National Science Foundation under Grant DMS-9626319 and by the U.S. Army Research Office under Contract DAAH04-95-1-0089. 


\title{
A new factorization technique of the matrix mask of univariate refinable functions
}

\author{
Gerlind Plonka \& Amos Ron
}

\section{Introduction}

\subsection{Factorization: general}

Let $\Phi$ be a finite subset of $L_{2}:=L_{2}(\mathbb{R})$, which we also treat as a vector with $n \in \mathbb{N}$ entries. Let $S(\Phi)$ be the smallest closed subspace of $L_{2}$ that contains each $E^{\alpha} \phi, \phi \in \Phi, \alpha \in \mathbb{Z}$. Here, $E^{\alpha}$ is the shift operator

$$
E^{\alpha}: f \mapsto f(\cdot+\alpha) .
$$

The space $S(\Phi)$ is known as a finitely generated shift-invariant (FSI, for short) space. FSI spaces are employed in several different areas of analysis, the most relevant ones to the present paper are wavelets and uniform subdivisions.

For certain applications concerning FSI spaces (one of which is discussed in $\$ 5$ of this paper), it is useful to attempt factoring the vector $\Phi$. Here, a factorization means expressing $\Phi$ in the form

$$
\Phi=V F,
$$

with $F$ a 'simpler' (in a suitable sense) vector, and $V$ a convolution operator, i.e., a matrix of order $\Phi \times F$, whose entries are convolution operators.

In more than one variable there are no universal factorization techniques (and the reasons for that are intrinsic), and this is the main reason the current paper is restricted to the more favorable univariate case. In that case, general factorization techniques can be either based on the dependence relations among the shifts of $\Phi$ or on the approximation properties of $S(\Phi)$. For example, assume that $\Phi=\{\phi\}$ is a singleton compactly supported distribution, and that, for some integer $k$,

$$
\phi *^{\prime} p:=\sum_{\alpha \in \mathbb{Z}} p(\alpha) E^{-\alpha} \phi \in \Pi, \quad \forall p \in \Pi_{k},
$$

where

is the space of all polynomials in one variable, and

$$
\Pi_{k}:=\{p \in \Pi: \operatorname{deg} p \leq k\}
$$

Then it is known, [R1], that $\phi$ can be written in the form $B * f$, with $B$ the B-spline of order $k$, and $f$ some distribution of shorter support. We will actually prove and invoke a stronger variant of that result in the current article.

A naive extension of the aforementioned result to the vector case is the following ansatz: "if the shifts of the compactly supported $\Phi$ span all polynomials of degree $\leq k$, then $\Phi=B * F$, with $B$ as before, and $F$ another compactly supported vector'. While this 'naive extension' is invalid, it is pleasing that a slightly modified version of it is true; however, the simple argument of [R1] does not carry over, and one needs to resort to some of the most powerful techniques in FSI space theory, techniques that nowadays are nicknamed superfunction theory. 
We are ready now to elaborate further on these matters. First (and in contrast with [R1]), our sole interest here is in a vector $\Phi$ which is refinable. By definition, this means that, for some dilation operator $\mathcal{D}_{s}$ of the form

$$
\mathcal{D}_{s}: f \mapsto f(\cdot / s),
$$

we have that

$$
\mathcal{D}_{s}(S(\Phi)) \subset S(\Phi)
$$

We will always assume that the dilation parameter $s$ is an integer greater than 1 . (The symbol $f(\cdot / s)$ above should be understood as the function $t \mapsto f(t / s))$. The refinability assumption on $S(\Phi)$ is equivalent, [BDR2], to the existence of a matrix $\mathbf{P}$ whose rows and columns are indexed by $\Phi$, and whose entries are measurable $2 \pi$-periodic functions, for which

$$
\widehat{\Phi}(s \cdot)=\mathbf{P} \widehat{\Phi}, \text { a.e. }
$$

The relation expressed in (1.2) is known as the refinement equation, and the matrix $\mathbf{P}$ is referred to as the mask symbol (or, sometimes, as the 'mask' or the 'symbol').

Certain guidelines should be followed when considering the problem of factoring a refinable $\Phi$. First, we require that each one of the convolution factors is refinable (note that B-splines are refinable with respect to all integer dilation parameters $s$ ). Second, in many practical situations the vector $\Phi$ is not explicitly known and the actual input is the symbol $\mathbf{P}$. Naturally, one then needs to factor the symbol $\mathbf{P}$, i.e., if the obtained factorization is, e.g., $\Phi=B * F$, then the practical information we seek is the mask symbol of $F$. The entire factorization can then be viewed as a factorization of $\mathbf{P}$; we will explain in the sequel the simple connection between the factorization at the function level and at the symbol level. Third, the functions $\Phi$ and/or the symbol $\mathbf{P}$ usually possess some 'favorable' properties. For example, the entries of $\mathbf{P}$ may be trigonometric polynomials, the functions in $\Phi$ may be compactly supported, their shifts may form a Riesz basis for $S(\Phi)$, etc. It is then desired that the process retains those properties, e.g., that the entries of the symbol $\mathbf{P}_{0}$ of the factor $F$ will still be polynomials, if those of $\mathbf{P}$ were ones.

In view of the above, we formalize the notion of 'factoring the mask symbol of a refinable function' as follows:

Definition 1.3. A $k$ th order factorization process is a process that applies to a class of 'admissible' refinable vectors $\Phi$. The input of the process is the mask symbol $\mathbf{P}$ of $\Phi$ and the output is a matrix $\mathbf{Q}$ such that $\mathbf{Q}$ is the mask symbol of a vector $F$ (whose cardinality equals that of $\Phi)$ for which $S\left(B_{k} * F\right)=S(\Phi)$, with $B_{k}$ the B-spline of order $k$.

Example: trivial factorization. The simplest factorization of order $k$ is the $k$-fold differentiation, i.e., $F:=D^{k} \Phi$. In that event, $B_{k} * F=\nabla^{k} \Phi$, with $\nabla$ the $k$ th-order backward difference, hence (since $S(\nabla G)=S(G)$ for any finite function set $\left.G \subset L_{2}\right)$ it is a 'factorization of order $k$ '. However, the relation $\mathbf{Q}=s^{k} \mathbf{P}$ clearly indicates that this factorization is practically useless. We will therefore impose in the sequel an additional condition on the factorization process, which will exclude the present trivial factorization.

\subsection{Superfunction theory and the factorization of $[\mathrm{P}]$}

The adjective "new' in the title of this paper certainly indicates that factorization techniques of refinable $\Phi$ 's are already in the literature. Indeed, a method for factoring the symbol of a refinable vector was introduced by the first named author in $[\mathrm{P}]$. In this subsection, we will first survey some aspects of superfunction theory, and recast the factorization of $[\mathrm{P}]$ in these terms. 
Superfunction theory is a notion intimately related to approximation orders of FSI spaces (the discussion here equally applies to multivariate functions; in fact, superfunction theory was motivated, first and foremost, by multivariate approximation problems). One says that the FSI space $S(\Phi)$ provides approximation order $k$ if, given any 'sufficiently smooth' function $g \in L_{2}$, we have that (as $h \rightarrow 0$ )

$$
\operatorname{dist}\left(g, \mathcal{D}_{h}(S(\Phi))\right)=O\left(h^{k}\right) .
$$

It was conjectured by Babuška (cf. [SF]) that if $\Phi$ is compactly supported (the FSI space is then termed local), and if $S(\Phi)$ provides approximation order $k$, then there exists a finite linear combination $\psi$ of $\Phi$ and its shifts (necessarily, thus, of compact support), such that the small subspace $S(\psi)$ of $S(\Phi)$ already provides the same approximation order $k$. Babuška's conjecture was proved true in [BDR2], and $\psi$ above (which, we emphasize, is not unique) was subsequently nicknamed 'superfunction'. The reference [BDR2] contains extensions of the aforementioned results to non-local FSI spaces; nonetheless, all those results are too general for our context: their ultimate success is their validity under extremely mild conditions, alas, the superfunctions, under those conditions, lack certain properties which we need here.

We will use instead the more recent (and also more straightforward) superfunction results that were established in [BDR4]. There, improved superfunction results were obtained under additional, reasonably mild, conditions on the vector $\Phi$. The basic condition there was termed Strong $H(k)$ Condition, and a slightly stronger (unnamed) condition was used there in the applications to refinable functions. We will recall these two conditions, and name the latter one. But, first, we recall the notions of the bracket product and the Gramian from [BDR1,BDR2].

Definitions 1.4 .

(a) Let $f, g \in L_{2}$. Their bracket product $[f, g]$ is the $2 \pi$-periodization of $f \bar{g}$ :

$$
[f, g]:=\sum_{l \in 2 \pi \mathbb{Z}} f(\cdot+l) \overline{g(\cdot+l)}
$$

Note that the bracket product lies in $L_{1}(\mathrm{TT})$, where TI denotes the torus.

(b) Let $\Phi \subset L_{2}$ be finite. The Gramian $\mathbf{G}:=\mathbf{G}_{\Phi}$ is the Hermitian $\Phi \times \Phi$ matrix whose $(\phi, \varphi)$ entry, $\phi, \varphi \in \Phi$, is the bracket product $[\widehat{\phi}, \widehat{\varphi}]$.

Definition 1.5: The strong $\mathrm{H}(\mathrm{k})$ property. Let $\Xi$ be a finite subset of $\mathbb{R}$, and let $k$ be a positive integer. We say that $\Phi$ satisfies the strong $\mathbf{H}(\mathrm{k})$ property at $\Xi$ if the following two conditions hold:

(I) For some neighborhood $O$ of the origin, and for each $\xi \in \Xi$, each $\widehat{\phi}, \phi \in \Phi$, as well as each entry of the Gramian $\mathbf{G}_{\Phi}$, is $k$ times continuously differentiable on $\xi+O+2 \pi \mathbf{Z}$.

(II) $\mathbf{G}(\xi)$ is invertible, for every $\xi \in \Xi$.

The default set $\Xi$ is $\{0\}$, thus the strong $\mathbf{H}(\mathrm{k})$ property means 'the strong $H(k)$ property at $\Xi=\{0\}$.

The unnamed condition in [BDR4] corresponds now to the strong $\mathrm{H}(\mathrm{k})$ property at

$$
\Xi_{s}:=\left\{\frac{2 \pi j}{s}: j=0, \ldots, s-1\right\},
$$

and this is indeed the basic condition we will need in this paper. It is worth stressing that this requirement is much weaker than more commonly used assumptions, such as the $L_{2}$-stability of the shifts of $\Phi$ (which is also known as 'the Riesz basis property') or the linear independence of those shifts. We elaborate on that point in the next section. 
We want now to explain the factorization technique that was developed in [P]. For that, we use the following univariate case of Theorem 4.2 of [BDR4]. The reader should immediately note that this is 'a superfunction result'. In this result, as elsewhere in this paper, the notation

stands for the differentiation operator.

Result 1.6. Assume that $\Phi$ satisfies the strong $H(k)$ property for some $k$, and that $S(\Phi)$ provides approximation order $k$. Then there exist vectors $y_{l}:=\left\{y_{l}(\phi): \phi \in \Phi\right\} \in \mathbb{C}^{\Phi}, l=0, \ldots, k-1$, such that $y_{0} \neq 0$, and such that, if $\psi$ is a finite linear combination of $\Phi$ and its shifts, i.e., if

$$
\widehat{\psi}=\sum_{\phi \in \Phi} \tau_{\phi} \widehat{\phi},
$$

for some trigonometric polynomials $\tau_{\phi}, \phi \in \Phi$, then $S(\psi)$ provides approximation order $j \leq k$ if

$$
D^{l} \tau_{\phi}(0)=y_{l}(\phi), \quad \phi \in \Phi, l=0, \ldots, j-1 .
$$

The factorization method of $[\mathrm{P}]$ revisited. This method is inductive. Assume that $\Phi$ satisfies the strong $\mathrm{H}(1)$ property, and that $S(\Phi)$ provides approximation order $k \geq 1$. Then, Result 1.6, a superfunction $\psi_{1}$ that provides approximation order 1 is available in the form

$$
\psi_{1}=\sum_{\phi \in \Phi} y_{0}(\phi) \phi .
$$

Now, order $\Phi$ in any way $\left\{\phi_{1}, \phi_{2}, \ldots, \phi_{n}\right\}$ so that, for some $j, y_{0}\left(\phi_{l}\right) \neq 0$ if and only if $l \leq j$. Then, replace the vector $\Phi=\left\{\phi_{1}, \phi_{2}, \ldots, \phi_{n}\right\}$ by a vector $\Psi=\left\{\psi_{1}, \psi_{2}, \ldots, \psi_{n}\right\}$, as follows:

(a) $\psi_{1}$ is the aforementioned superfunction.

(b) For $l=1, \ldots, j-1, \psi_{l+1}=\psi_{l}+y_{0}\left(\phi_{l}\right) \nabla \phi_{l}$, with $\nabla$ the backward difference operator $\nabla=$ $1-E^{-1}$.

(c) For $l>j, \psi_{l}=\nabla \phi_{l}$.

Obviously, $S(\Psi)=S(\Phi)$. Under a mild condition on $\psi_{1}$ (e.g., a mild decay condition at $\infty$ ), the fact that $\psi_{1}$ provides approximation order 1 implies that $\psi_{1}=B_{1} * f_{1}$, with $B_{1}$ the B-spline of order 1 , and $f_{1}$ some function/distribution. Also, for every function $g, \nabla g=B_{1} * D g$. All in all, one concludes that there exists a vector $F$ (consisting of either functions or distributions), such that

$$
\Psi=B_{1} * F .
$$

If $F$ lies in $L_{2}$, one can show that it provides approximation order $k-1$. If it still satisfies the $\mathrm{H}(1)$ property, the process may be continued, and another factorization step can be applied.

The above description does not mention refinability. In contrast, the process in [P] assumes refinability and is described there as an algorithm for factoring the mask symbol. Indeed, the above vector $F$ is still refinable, and the highlight of the factorization of $[\mathrm{P}]$ is the (non-trivial) fact that, if the original symbol $\mathbf{P}$ of $\Phi$ consists of trigonometric polynomials, so is the symbol of the new $F$ (cf. [P] for more details. The strong $\mathrm{H}(1)$ property does not suffice for the validity of the last assertion: one needs for that to assume the strong $\mathrm{H}(1)$ property at $\Xi_{s}$. $[\mathrm{P}]$ makes an assumption that is equivalent to $L_{2}$-stability.)

While the above details correspond exactly to the algorithm in $[\mathrm{P}]$ (with the only difference being that we use here backward difference as opposed to the forward difference used in [P]), it should be stressed that many other variants of that algorithm are possible. One should only follow the rule that $\psi_{j}$ above, $j>1$, lies in the span of $\left\{\psi_{1}, \nabla \phi_{1}, \nabla \phi_{2}, \nabla \phi_{3}, \ldots\right\}$, with $\psi_{1}$ the aforementioned superfunction. 


\subsection{Our new factorization technique: why and what?}

The method of this article is developed under assumptions weaker than those assumed in [P]. However, it will be erroneous to conclude that our aim is at a factorization method that applies to a wider range of FSI spaces. Had we wanted to, we could have rederived the method of $[\mathrm{P}]$ under the conditions we assume here.

In order to explain our motivation, we first note that the factorization outlined in the previous subsection 'shuffles' the vector $\Phi$. More precisely, in contrast with the unavailable 'naive factorization' $\Phi=B * F$ (where there is a natural correspondence between the elements of $\Phi$ and $F$ ), no simple relation exists (in the method of $[\mathrm{P}]$ ) between the original entries of $\Phi$ and those of the factor $F$. For the application of certain emerging algorithms this is a disadvantage. Specifically, in [RS] a new technique for estimating the smoothness of refinable functions was developed. The technique there does not require the factorization of the mask symbol, but its numerical stability is dramatically improved if a factorization is first done. On the other hand, the [RS] method allows one to find separately the smoothness of each entry of $F$ (previous results, such as that of [CDP] which incidentally is based on the [P] factorization, could find only the common smoothness of all the functions in $\Phi$ ). However, as we had just explained, the existing factorization techniques 'scramble' the functions in $\Phi$, hence one cannot convert the componentwise smoothness estimates on $F$ to componentwise smoothness estimates on $\Phi$. This drawback is exactly the one that we overcome with the new method: in this new method, there will be a simple correspondence between the functions in the original $\Phi$ and those in the final factor $F$.

Our method can start with a vector $\Phi$ that may or may not be refinable. Assuming $S(\Phi)$ to provide approximation order $k$, we attempt to replace one $\phi_{0} \in \Phi$ by a superfunction $\psi$ (whose $S(\psi)$ also provides the same approximation order), and to further replace each the remaining $\phi \in \Phi$ by $\nabla^{k} \phi$, where, as before, $\nabla$ is the backward difference. Regardless of the choice of $\psi$, the resulting vector $\Phi_{0}$ is factorable into the form $\Phi_{0}=B_{k} * F$ (with $B_{k}$ the B-spline of order $k$ ). The factorization is adequate: for example, if $\Phi$ are of compact support, so are $F$. However, more care is required upon assuming $\Phi$ to be refinable. For example, if the symbol of $\Phi$ is made of trigonometric polynomials, $F$ is refinable, too, but its symbol, in general, will not be made of polynomials. This is the reason that we rely on subtle superfunction results: the crux is to find a superfunction $\psi$ so that the polynomiality of the symbol is retained when replacing $\phi_{0}$ by $\psi$. Theorem 4.12 and Corollary 5.5 of [BDR4] establish the existence of such superfunction $\psi$, indicate which entry $\phi_{0}$ of $\Phi$ can be replaced by $\psi$, and compute the symbol of the new $\Phi_{0}$. (As a matter of fact, the [BDR4] results we use were tailored by the [BDR4] authors to help us in developing the present method.) We review those details in $\S 2$, and also prove in that section the extension of the [R1] result that guarantees the factorization of the superfunction $\psi$ into the form $B_{k} * F$.

In $\S 3$, we provide a unified approach to factorization which is based on superfunction theory basics. In $\S 4$, we discuss the theoretical aspects of our factorization, and then use the results of $\S 3$ in the derivation of an algorith $m$ for the factorization process. In $\S 5$ we explain how the factorization can help in determining the smoothness of refinable vectors via the processing of its mask.

\section{Superfunctions, refinable functions, and other preliminaries}

We discuss here three topics in preparation for the factorization algorithm. First, we quote several superfunction results from [BDR4]; some are used in the next section in a general analysis of factorization, while others are needed in order to 'prepare' the vector $\Phi$ for our specific factorization. We also present a theorem that allows us to factor the B-spline from the superfunction (and which we already alluded to in the introduction). We finally discuss the notions of $L_{2}$-stability and pre-stability, notions that appear in the statements of our main results. 
As we already mentioned in $\S 1.2$, given an FSI space $S(\Phi)$ that provides approximation order $k$, one can find in $S(\Phi)$ many superfunctions. The study of [BDR4] focuses, initially, on a canonically chosen superfunction $\psi_{0}$ which, in the univariate case, has the form

$$
\widehat{\psi}_{0}=\sum_{\phi \in \Phi} \sigma_{\phi} \widehat{\phi}
$$

where $\sigma_{\phi}$ is a polynomial in the span of $\left\{e^{-i j u}: j=0,1, \ldots, k-1\right\}$, and which satisfies $D^{j} \widehat{\psi}_{0}(l)=$ $\delta_{l, 0} \delta_{j, 0}(l \in 2 \pi \mathbb{Z}, 0 \leq j \leq k-1)$. We label this particular superfunction the canonical superfunction (of order $k$ ). Theorem 4.2 of [BDR4] proves that, under the strong $\mathrm{H}(\mathrm{k})$ property, there exists a unique canonical superfunction. The trigonometric polynomial row vector

$$
\sigma:=\left(\sigma_{\phi}\right)_{\phi \in \Phi}
$$

of (2.1) will be referred to as the canonical $\Phi$-vector of order $k$. As we will see in the sequel, the canonical vector provides key information in the implementation of factorization algorithms, and therefore, it is important to be able to compute that vector. In [BDR4], two different methods are suggested to that end: one (cf. Corollary 4.9 there) does not assume refinability, and relies on values of the derivatives of $\widehat{\Phi}$ at $2 \pi \mathrm{ZZ}$. The other one (cf. Theorem 5.2 there) extracts the canonical vector directly from the mask symbol $\mathbf{P}$ of the refinable $\Phi$, and reads, for a univariate setup, as follows:

Result 2.2. Let $\Phi \subset L_{2}$ be s-refinable with mask symbol P. Suppose that $\Phi$ satisfies the strong $H(k)$ property at $\Xi_{s}:=\frac{2 \pi}{s}\{0,1, \ldots, s-1\}$. Then the following conditions are equivalent:

(a) $S(\Phi)$ provides approximation order $k$.

(b) There exists a trigonometric polynomial row vector $\sigma=\left(\sigma_{\phi}\right)_{\phi \in \Phi}$, so that,

(i) $\sigma(0) \widehat{\Phi}(0)=1$,

(ii) $\sigma(s \cdot) \mathbf{P}$ has a $k$-fold zero at each of $\Xi_{s} \backslash 0$, and $\sigma(s \cdot) \mathbf{P}-\sigma$ has a $k$-fold zero at the origin:

$$
D^{j}\left(\sigma(s \cdot) \mathbf{P}-\delta_{\xi, 0} \sigma\right)_{u=\xi}=0, \quad \xi \in \Xi_{s}, \quad j=0, \ldots, k-1 .
$$

Moreover, $\sigma$ in (b) is the (unique) canonical $\Phi$-vector of order $k$, provided each $\sigma_{\phi}$ lies in the span of $\left(e^{-i j u}\right)_{j=0}^{k-1}$. Finally, the implication $(b) \Longrightarrow$ (a) holds under a weaker assumption, viz., that $\Phi$ satisfies the strong $H(k)$ property at 0 only.

Proof: $\quad$ The fact that (a) $\Longrightarrow$ (b) follows from Theorem 5.2 of [BDR4]: the theorem there states that, if $S(\Phi)$ provides approximation order $k$ and if $\Phi$ satisfies the strong $H(k)$ property, then (b) holds for some vector $\sigma$. That vector $\sigma$ can be chosen as the canonical vector, and this choice is indeed made in the proof in [BDR4] for Theorem 5.2.

The converse implication, (b) $\Longrightarrow(\mathrm{a})$, is also contained in the proof of Theorem 5.2 of [BDR4]: the proof there shows that, assuming (b), the function $\psi_{0}$ defined by $\widehat{\psi}_{0}=\sigma \widehat{\Phi}$ is the canonical superfunction of order $k$.

However, for the specific factorization technique of $\S 4$, we need a further superfunction result. That other superfunction result is Theorem 4.12 of [BDR4] that reads in the univariate case as follows:

Result 2.3. Let $k$ be a positive integer, and let $\Phi$ be a vector of $L_{2}$-functions satisfying the strong $H(k)$ property. If $S(\Phi)$ provides approximation order $k$, then there exists $\phi_{0} \in \Phi$ and for this $\phi_{0}$ a unique function $\psi$ that satisfies the following two properties: 
(a) $\psi$ satisfies on the Fourier domain a relation of the form

$$
\widehat{\psi}=\widehat{\phi}_{0}+\sum_{\phi \in\left(\Phi \backslash \phi_{0}\right)} \tau_{\phi} \widehat{\phi}
$$

with each $\tau_{\phi}$ a trigonometric polynomial in the span of $\left(e^{-i j u}\right)_{j=0}^{k-1}$.

(b) $\psi$ satisfies the Strang-Fix conditions of order $k$ :

$$
\widehat{\psi}(0) \neq 0, \quad D^{j} \widehat{\psi}(l)=0 \quad(j=0, \ldots,, k-1, l \in 2 \pi \mathbb{Z} \backslash\{0\}) .
$$

Moreover, $S(\psi)$ provides a pproximation order $k$.

Corollary 5.5 of [BDR4] demonstrates the usefulness of this superfunction in the context of refinable vectors. We quote below the univariate case of that corollary, and provide, for completeness, its simple proof. More details concerning the above result are found in $\S 4$, where we discuss a method for finding this special superfunction; more precisely, we will need to find the trigonometric polynomials $\left(\tau_{\phi}\right)$ above. The computation of $\tau_{\phi}$ will be based on the canonical $\Phi$-vector $\sigma$ of order $k$ in Result 2.2. For convenience, we set

$$
\Phi^{\prime}:=\Phi \backslash \phi_{0},
$$

and

$$
\Phi_{0}:=\Phi^{\prime} \cup \psi
$$

with $\phi_{0}$ and $\psi$ as in Result 2.3.

Result 2.5. Assume that the assumptions, hence the conclusions, of Result 2.3 hold. Assume further that the vector $\Phi$ is refinable with mask symbol $\mathbf{P}$. Then, in the notations of Result 2.3, $\Phi_{0}$ (defined in (2.4)) is also refinable with a symbol $\mathbf{P}_{0}$ of the form

$$
\mathbf{P}_{0}=\mathbf{U}_{1}(s \cdot) \mathbf{P} \mathbf{U}_{2},
$$

where the entries of $\mathbf{U}_{1}, \mathbf{U}_{2}$ are trigonometric polynomials. More precisely, if we order the functions in $\Phi_{0}$ in any order of the form $\left(\psi, \phi_{2}, \phi_{3}, \ldots\right)$, then $\mathbf{U}_{1}=\mathbf{I}+\mathbf{R}, \mathbf{U}_{2}=\mathbf{I}-\mathbf{R}$, with $\mathbf{R}$ a matrix whose first row is

$$
\left(0, \tau_{\phi_{2}}, \tau_{\phi_{3}}, \ldots\right)
$$

and all its other rows 0 (and with $\tau_{\phi}$ the trigonometric polynomials of Result 2.3).

Proof: Let $\psi$ be the superfunction of Result 2.3, its existence and uniqueness has been shown in [BDR4], Theorem 4.12. It is evident that the connection between the vectors $\Phi$ and $\Phi_{0}$ is, on the Fourier domain, of the form

$$
\widehat{\Phi}_{0}=\mathbf{U}_{1} \widehat{\Phi}
$$

with $\mathbf{U}_{1}$ having the structure stated in the result. Thus, the symbol $\mathbf{P}_{0}$ of $\Phi_{0}$ is

$$
\mathbf{P}_{0}=\mathbf{U}_{1}(s \cdot) \mathbf{P U}_{1}^{-1} .
$$

It is straightforward to check that $\mathbf{U}_{1}^{-1}=\mathbf{U}_{2}$, with $\mathbf{U}_{2}$ as in statement of the result. 
We postpone the discussion concerning the computation of $\psi$ as well as explicit constructions of $\mathbf{U}_{1}, \mathbf{U}_{2}$ to $\S 4$.

Almost all the observations that are required for the new factorization hold in several variables (though they are stated and proved in one dimension). The only result that does not extend beyond the univariate setup is the next theorem. As mentioned before, the compact support case of this result (i.e., statement (a) here) can already be found in [R1].

Theorem 2.6. Let $\psi \in L_{2}(\mathbb{R})$ and assume that, for some origin neighborhood $O, \widehat{\psi}$ is $k$-times continuously differentiable on $O+2 \pi \mathbb{Z} \backslash 0$, and is bounded on $O$. If $S(\psi)$ provides approximation order $k$, and if $D^{k} \widehat{\psi}$ grows slowly on $O+2 \pi Z \backslash \backslash$, then there exists a tempered distribution $\eta$ such that $\psi=B_{k} * \eta$, with $B_{k}$ the $B$-spline of order $k$. Moreover, $\eta$ can be chosen in a way that:

(a) If $\operatorname{supp} \psi \subset[a, b]$, then $\operatorname{supp} \eta \subset[a, b-k]$.

(b) If $D^{k} \psi(u)=O\left(|u|^{-\rho}\right)$ for large $|u|$, and for some $\rho>k+\varepsilon$, then $\eta(u)=O\left(|u|^{-(\rho-k)}\right)$.

(c) If $D^{k} \psi(u)=O\left(e^{-\beta|u|}\right)$, for some positive $\beta$, then $\eta$ decays at that same exponential rate.

Proof: $\quad$ First, since we assume that $S(\psi)$ provides approximation order $k$, and since we further assume that $\widehat{\psi}$ is bounded around the origin, then, $\psi$ must have a $k$-fold zero at each $j \in 2 \pi \mathbb{Z} \backslash 0$ (see [BDR1]).

Let $G_{1}$ be the support function of $O+2 \pi Z Z, G_{2}:=1-G_{1}$. We consider the expression (with $\left.B:=B_{k}\right)$

$$
\frac{\widehat{\psi}}{\widehat{B}}=\frac{G_{1} \widehat{\psi}}{\widehat{B}}+\frac{G_{2} \widehat{\psi}}{\widehat{B}} .
$$

We will show that each of the two summands above is a tempered distribution, and this will prove the existence of the required tempered distribution $\eta$ (we will then use other techniques to prove the decay assertions).

Firstly, recall that

$$
\widehat{B}(u)=\left(\frac{1-e^{-i u}}{i u}\right)^{k}
$$

Since $1 /\left(1-e^{-i u}\right)$ is bounded on $\operatorname{supp} G_{2}$, we then conclude that $G_{2} / \widehat{B}$ grows only slowly at $\infty$, hence the function $G_{2} \widehat{\psi} / \widehat{B}$ is a tempered distribution.

Secondly, let $j \in 2 \pi \mathbb{Z} \backslash 0$. We then write

$$
\widehat{\psi}(u) / \widehat{B}(u)=\frac{\widehat{\psi}(u)}{(u-j)^{k}}\left(\frac{u-j}{1-e^{-i u}}\right)^{k}(i u)^{k} .
$$

Note that $\left\|\left(\frac{u-j}{1-e^{-i u}}\right)^{k}\right\|_{L_{\infty}(j+O)}=\|1 / \widehat{B}\|_{L_{\infty}(j+O)} \leq$ const. Thus, since $\widehat{\psi}$ is assumed to be smooth at each region of the form $j+O, j \in 2 \pi \mathbb{Z} \backslash 0$ and has a $k$-fold zero at $j$, it follows that

$$
\left\|G_{1} \widehat{\psi} / \widehat{B}\right\|_{L_{\infty}(j+O)}=\|\widehat{\psi} / \widehat{B}\|_{L_{\infty}(j+O)} \leq \mathrm{const}|| D^{k} \widehat{\psi} \|_{L_{\infty}(j+O)}|j|^{k}
$$

Since we assume $D^{k} \widehat{\psi}$ to grow slowly on $O+2 \pi \mathbb{Z} \backslash 0$, we conclude from the above that $G_{1} \widehat{\psi} / \widehat{B}$ grows slowly, as well. This concludes the proof of the existence of $\eta$ satisfying $\psi=B * \eta$.

Now, we prove (a-c), and use for that a different argument (which applies uniformly well to all three cases, and which is an adaptation of the argument in [R1]). Firstly, note that the Fourier transform of $D^{k} \psi$ has a $k$-fold zero at each $j \in 2 \pi \mathbb{Z} \backslash 0$, and also has a similar zero at the origin. Using any of the decay conditions (a-c), a standard application of Poisson's summation formula then yields that

$$
\left(D^{k} \psi\right) *^{\prime} p=0, \quad \forall p \in \Pi_{k-1} .
$$


We choose $p$ to be the polynomial of degree $k-1$ that makes the following identity valid

$$
\eta:=\sum_{\alpha=k}^{\infty} p(\alpha) E^{\alpha}\left(D^{k} \psi\right)=\sum_{\alpha_{1}=1}^{\infty} \cdots \sum_{\alpha_{k}=1}^{\infty} E^{\alpha_{1}+\ldots+\alpha_{k}}\left(D^{k} \psi\right)
$$

(i.e., $\left.\nabla^{k-1} p=(-1)^{k-1}\right)$. The decay assumption on $D^{k} \psi$ grants us that the sum that defines $\eta$ converges absolutely (distributionally). That same decay assumption can be easily used to show that, as $t \rightarrow \infty, \eta(t)$ decays as it is required to. However, since $\left(D^{k} \psi\right) *^{\prime} p=0$ (and since $p$ vanishes at $\{1, \ldots, k-1\})$, we have the alternative expression for $\eta$

$$
\eta=\sum_{\alpha=-\infty}^{0} p(\alpha) E^{\alpha}\left(D^{k} \psi\right)
$$

which can be used to show that $\eta$ also decays suitably at $-\infty$. Finally, the right-most expression for $\eta$ in (2.7) implies that $\nabla^{k} \eta=D^{k} \psi$. Now, set $g:=B * \eta$. Since $B$ is a compactly supported function, $g$ decays at $\infty$ at least at the same rate as $\eta$ does. Further, $D^{k} g=\nabla^{k} \eta$, which means that $D^{k}(g-\psi)=0$, hence that $g-\psi$ is a polynomial. That polynomial is 0 , since both $g$ and $\psi$ vanish at $\infty$.

We now turn our attention to the third topic of this section: stability and pre-stability. We recall that a subset $G \subset L_{2}\left(\mathbb{R}^{d}\right)$ is called $L_{2}$-stable if there exist two positive constants $c, C$, such that, for any finitely supported sequence $u$ defined on $G$,

$$
c\|u\|_{\ell_{2}(G)} \leq\left\|\sum_{g \in G} u(g) g\right\|_{L_{2}} \leq C\|u\|_{\ell_{2}(G)} .
$$

We are interested in the $L_{2}$-stability of the set $E(\Phi)$ of the integer shifts of $\Phi$. That stability property was characterized in [BDR2] as follows:

Result 2.8. Let $\Phi \subset L_{2}$ be finite with Gramian G. For each $\omega \in \mathbb{R}$, let $\Lambda(\omega)$ (respectively, $\lambda(\omega)$ ) be the largest (respectively, smallest) eigenvalue of $\mathbf{G}(\omega)$. Then the shifts $E(\Phi)$ of $\Phi$ are stable if and only if the functions $\Lambda$ and $1 / \lambda$ are essentially bounded on $[-\pi, \pi]$.

The above result is somewhat inconvenient for our purposes, since it does not allow a simple extension of the stability property from $L_{2}$-functions to distributions. Therefore we will use instead the following related notion, labeled 'pre-stability'.

Definition 2.9. Let $\Phi$ be a finite collection of tempered distributions and assume that $\widehat{\Phi}$ are continuous. We say that the shifts $E(\Phi)$ of $\Phi$ are pre-stable if, for each $\theta \in \mathbb{R}$, the restriction of $\widehat{\Phi}$ to $\theta+2 \pi \mathbb{Z}$ is linearly independent.

The following result draws a connection between the notions of stability and pre-stability:

Proposition 2.10. Let $\Phi \subset L_{2}$ be finite, and assume that for each $\phi \in \Phi$ (i) $\widehat{\phi}$ is continuous, and (ii) $[\widehat{\phi}, \widehat{\phi}]$ is finite everywhere. Let $\lambda$ be the eigenvalue function of Result 2.8. If $E(\Phi)$ are pre-stable, then $1 / \lambda \in L_{\infty}(\mathbb{R})$.

Proof of Proposition 2.10. We let $\mathbf{G}_{m}, m \in \mathbb{N}$, be the matrix obtained from the Gramian $\mathbf{G}$ of $\Phi$ by replacing each bracket product $[\widehat{\varphi}, \widehat{\phi}]$ with

$$
\sum_{j=-m}^{m} \hat{\varphi}(\cdot+2 \pi j) \overline{\widehat{\phi}}(\cdot+2 \pi j)
$$


Fix $\omega \in[-\pi, \pi]$, and let $\lambda_{m}(\omega)$ be the smallest eigenvalue of $\mathbf{G}_{m}(\omega)$. Since the entries of $\mathbf{G}(\omega)$ are assumed finite, $\mathbf{G}_{m}(\omega)$ converges to $\mathbf{G}(\omega)$, and hence

$$
0 \leq \lambda_{m}(\omega) \uparrow \lambda(\omega) .
$$

(since $\mathbf{G}(\omega), \mathbf{G}_{m}(\omega)$ as well as $\left(\mathbf{G}-\mathbf{G}_{m}\right)(\omega)$ are Hermitian and non-negative). Furthermore, since the entries of $\mathbf{G}_{m}$ are continuous, so is the function $\lambda_{m}$, hence its zero set $\Omega_{m} \subset[-\pi, \pi]$ is compact.

Now, the assumption of pre-stability is equivalent to $\lambda$ having no zeros. This means that $\cap_{m} \Omega_{m}=\emptyset$, hence that $\Omega_{m}=\emptyset$, for a large enough $m$. But this implies that the continuous function $\lambda_{m}$ vanishes nowhere, hence that $1 / \lambda_{m}$ is bounded; a fortiori $1 / \lambda$ is bounded.

Thus, if we know that $\Phi$ are in $L_{2}$, that their Fourier transform is continuous, and that their Gramian matrix has bounded entries, pre-stability implies stability. The boundedness of the Gram entries is a 'minor' assumption, in the sense that it is usually implied by technical conditions on $\Phi$ (either a mild decay condition on $\Phi$, or, in the case $\widehat{\Phi}$ is continuous, by a mild smoothness assumption on $\Phi)$. Moreover, the following result, which is essentially due to [JM], provides simple conditions under which pre-stability and stability become equivalent notions for $L_{2}$-vectors $\Phi$. One should keep in mind, however, that the main point in the pre-stability notion is its applicability to functions and distributions outside $L_{2}$.

Corollary 2.11. Let $\Phi \subset L_{2}$ be finite, and assume that the entries of the Gramian $\mathbf{G}$ of $\Phi$ are continuous (i.e., equal a.e. to continuous functions). Then the shifts of $E(\Phi)$ are stable if and only if they are pre-stable.

We note that the product $[\widehat{f}, \widehat{g}]$ is the Fourier series of the sequence

$$
\mathbb{Z} \ni j \mapsto\left\langle f, E^{j} g\right\rangle,
$$

hence that the product is continuous if, e.g., $f$ and $g$ are both $O\left(|\cdot|^{-(1+\varepsilon)}\right)$ at $\infty$, for some $\varepsilon>0$.

Proof: Since $\mathbf{G}$ has continuous entries, its eigenvalue function $\Lambda$ is continuous (and $2 \pi$ periodic). This implies that $\Lambda$ is bounded, hence that stability is equivalent here to the boundedness of $1 / \lambda$. Since $\lambda$ is continuous, too, $1 / \lambda$ is bounded iff $\lambda$ vanishes nowhere. That latter condition is equivalent to pre-stability.

The discussion so far reveals that the stability of $E(\Phi)$ can be roughly thought of as the invertibility of $\mathbf{G}_{\Phi}$ everywhere. At the same time, the essential requirement in the strong $\mathrm{H}(\mathrm{k})$ property at $\Xi$ 'is the invertibility of $\mathbf{G}_{\Phi}$ at each $\xi \in \Xi$. Thus, the $H(k)$ property is a significant relaxation of the stability requirement.

\section{General facts about factorizations}

Before we develop the new factorization technique that we aim at, we would like to clarify the notion of a 'factorization process'. We have already defined it in the introduction, but immediately showed there that, as defined, the notion is broad enough to admit 'factorizations' that are useless in nature. In this section, we provide further details about the nature of a 'useful factorization' and discuss the relations between those 'useful factorizations' on the one hand, and the matrices involved in the factorization on the other hand. The general discussion here will be used in the next section in the conversion of our new factorization technique from a mere existence theorem to an actual algorithm. 
The following example is the prototype of what may be considered a 'useful factorization', and is hidden in the core of all practical factorizations.

The simplest example of 'good' factorization. Assume that the vector $\Phi$ contains a function $\phi_{0}$ whose SI space $S\left(\phi_{0}\right)$ provides approximation order $k$. Then, under some mild conditions on $\phi_{0}$, Theorem 2.6 provides us with a factorization $\phi_{0}=B_{k} * \eta$, for some distribution $\eta$. We then define the factor $F$ to be $\left(\eta, D^{k}\left(\Phi \backslash \phi_{0}\right)\right)$. Note that $B_{k} * F=\left(\phi_{0}, \nabla^{k}\left(\Phi \backslash \phi_{0}\right)\right)=: \Phi_{1}$. Since (cf. e.g., [BDR1]) $S\left(\Phi_{1}\right)=S(\Phi)$, we conclude that the above process is indeed a 'factorization of order $k$ '.

\section{Remarks.}

(1) One should note that there is only a subtle difference between the example here and the trivial (and worthless) factorization ( $\$ 1.1$ ).

(2) The above example fully demonstrates the role played here by 'superfunction theory'. The function $\phi_{0}$ is clearly a superfunction. If the original vector $\Phi$ does not contain a superfunction, the factorization process starts with changing the vector $\Phi$ to another vector, say $\Phi_{1}$, which contains a superfunction among its entries. If the symbol $\mathbf{P}$ of $\Phi$ is known to be a polynomial matrix, then the actual challenge here is to select $\Phi_{1}$ such that its symbol is a polynomial matrix, too. The move then from the symbol of $\Phi_{1}$ to the symbol of $F$ is guaranteed to preserve the polynomiality of the mask symbol.

Questions addressed in this section: A factorization was defined as $\Phi \mapsto F$, with $S(\Phi)=$ $S\left(B_{k} * F\right)$. This yields the existence of a matrix $\mathbf{C}$ with $2 \pi$-periodic measurable entries such that, with $\mathbf{P}$ and $\mathbf{Q}$ the mask symbols of $\Phi$ and $F$ respectively,

$$
\mathbf{P C}=s^{-k} \mathbf{C}(s \cdot) \mathbf{Q}, \quad \text { a.e. }
$$

(See assertion (a) in the theorem below.) We are then interested here in the following two questions:

(1) How exactly we distinguish between 'useful' and 'useless' factorizations?

(2) What are the characteristics of the transition matrices $\mathbf{C}$ that are involved in 'useful' factorizations?

Definition: good factorizations. A good factorization process of order $k$ is a factorization process of order $k \Phi \mapsto F$ (i.e., $S(\Phi)=S\left(B_{k} * F\right)$ ) such that $\widehat{F}$ is continuous at 0 and $\widehat{F}(0) \neq 0$.

The following theorem establishes the connection between good factorizations and their corresponding transition matrices. Connections are also drawn between those transition matrices and the superfunctions in the FSI space. A complementary theorem (Theorem 3.6) discusses the converse problem, of choosing the transition matrix $\mathbf{C}$ in a way that, given a matrix $\mathbf{Q}$, the 'lifted' matrix $\mathbf{P}$ (defined by $\mathbf{P C}=s^{-k} \mathbf{C}(s \cdot) \mathbf{Q}$ ) is the symbol of a vector $\Phi$ whose FSI space provides approximation order $k$.

Theorem 3.2. Let $\Phi \mapsto F$ be a factorization process of order $k$, and assume that $\widehat{\Phi}$ is bounded around the origin and that $\widehat{F}$ is continuous at each $j \in 2 \pi \mathbb{Z}$. Then:

(a) $\Phi$ is refinable if and only if $F$ is refinable. Furthermore, the corresponding mask symbols $\mathbf{P}$ and $\mathbf{Q}$ satisfy a relation

$$
\mathbf{P C}=s^{-k} \mathbf{C}(s \cdot) \mathbf{Q}
$$

for some matrix $\mathbf{C}$ with $2 \pi$-periodic entries. In particular,

$$
\widehat{\Phi}(u)=\frac{1}{(i u)^{k}} \mathbf{C}(u) \widehat{F}(u) .
$$


(b) If $F$ and $\Phi$ are refinable with symbols $\mathbf{P}$ and $\mathbf{Q}$, then the determinant of the matrix $\mathbf{C}$ in the relation $\mathbf{P C}=s^{-k} \mathbf{C}(s \cdot) \mathbf{Q}$ must have a $k$-fold zero at the origin, provided that the factorization is good, and that the entries of $\mathbf{C}$ are smooth at the origin.

(c) Assume, in addition to (b), that $\widehat{\Phi}$ are linearly independent over $2 \pi \mathbb{Z Z}$. Let $\psi$ be a function in $S(\Phi): \widehat{\psi}=\tau \widehat{\Phi}$, with $\tau$ a row vector indexed by $\Phi$ whose entries are bounded $2 \pi$-periodic, and are smooth at the origin. If $\psi$ is a superfunction (i.e., $S(\psi)$ provides approximation order $k$ ) then $\tau \mathbf{C}$ has a $k$-fold zero at the origin.

Most of the technical conditions that are assumed in the above theorem are satisfied automatically in practical situations. For example, the following corollary is a special case of Theorem 3.2 :

Corollary 3.3. Let $\Phi \mapsto F$ be a good factorization process of order $k$. Assume that $\Phi$ and $F$ are compactly supported refinable vectors with polynomial refinement symbols $\mathbf{P}$ and $\mathbf{Q}$ which satisfy the relation $\mathbf{P C}=s^{-k} \mathbf{C}(s \cdot) \mathbf{Q}$, for some trigonometric polynomial matrix $\mathbf{C}$. Then:

(a) $\operatorname{det} \mathbf{C}$ vanishes to order $k$ at the origin.

(b) Assume $\widehat{\Phi}$ are linearly independent over $2 \pi \mathbb{Z}$, and let $\tau=\left(\tau_{\phi}\right)_{\phi \in \Phi}$ be a vector of trigonometric polynomials. If the compactly supported function $\psi$ defined by

$$
\widehat{\psi}:=\tau \widehat{\Phi}
$$

is a superfunction, then $\tau \mathbf{C}$ vanishes at the origin to order $k$.

Proof of Theorem 3.2. (a) Since we assume that $S(\Phi)=S\left(B_{k} * F\right)$, there exist, [BDR2], an a.e. invertible matrix $\mathbf{U}$ with $2 \pi$-periodic measurable entries such that

$$
\widehat{\Phi}=\widehat{B_{k}} \mathbf{U} \widehat{F} .
$$

If $\widehat{\Phi}$ is refinable with mask symbol $\mathbf{P}$, we conclude from

$$
\widehat{B_{k}}(s \cdot) \mathbf{U}(s \cdot) \widehat{F}(s \cdot)=\widehat{\Phi}(s \cdot)=\mathbf{P} \widehat{\Phi}=\widehat{B_{k}} \mathbf{P} \mathbf{U} \widehat{F}
$$

that $F$ is refinable with symbol $\mathbf{Q}=\frac{\widehat{B_{k}}}{\widehat{B_{k}}(s \cdot)} \mathbf{U}^{-1}(s \cdot) \mathbf{P} \mathbf{U}$. Analogous computations prove that $\Phi$ is refinable whenever $F$ is refinable. Thus, (a) is established with $\mathbf{C}:=\left(1-e^{-i \cdot}\right)^{k} \mathbf{U}$.

(b): From the relation (3.1) (which was established in (a)), and the fact that $D^{k} \Phi$ is refinable with symbol $\mathbf{P}^{\prime}:=s^{k} \mathbf{P}$, we conclude that $\mathbf{P}^{\prime} \mathbf{C}=\mathbf{C}(s \cdot) \mathbf{Q}$, hence that $\widehat{D^{k}} \Phi=\mathbf{C} \widehat{F}$. Since $\widehat{F}(0) \neq 0$, we may assume that the first entry of $\widehat{F}(0)$ is non-zero. Let $\mathcal{F}$ be the matrix obtained from the identity matrix by replacing its first column by $\widehat{F}$. Then $\operatorname{det} \mathcal{F}$ is continuous at the origin and does not vanish there. On the other hand, $\operatorname{det} \mathbf{C} \operatorname{det} \mathcal{F}$ vanishes at the origin to order $k$ since $\mathbf{C} \mathcal{F}$ contains the column $\widehat{D^{k}} \Phi$ which vanishes to that order at the origin. Thus, det $\mathbf{C}$ has a $k$-fold zero at the origin.

(c): Since $\widehat{\psi}=\tau \widehat{\Phi}$ is a superfunction, and since $\tau$ is bounded while $\widehat{\Phi}$ is bounded around the origin, it follows (cf. [BDR1: Theorem 1.14]) that $\widehat{\psi}$ has a $k$-fold zero at each $j \in 2 \pi \mathbb{Z} \backslash 0$, hence that $\widehat{D^{k}} \psi$ has such a zero everywhere on $2 \pi \mathbb{Z}$. On the other hand $\widehat{D^{k}} \psi=\tau \widehat{D^{k} \Phi}=\tau \mathbf{C} \widehat{F}$, with the second equality following from the proof of (b). But $\tau \mathbf{C}$ is $2 \pi$-periodic, and the vectors $\widehat{F}(j)$, $j \in 2 \pi \mathbb{Z}$, span all of $\mathbb{C}^{F}$ (due to the linear independence of $\widehat{F}$ over $2 \pi \mathbb{Z}$ ), hence it easily follows that $\tau \mathbf{C}$ vanishes to order $k$ at the origin. 
Example: Orthogonal GHM-scaling functions [GHM]. Choose $s=n=2$, and let $\Phi=$ $\left(\phi_{1}, \phi_{2}\right)^{T}$ be the vector which has the mask symbol

$$
\mathbf{P}(u)=\frac{1}{20}\left(\begin{array}{cc}
6\left(1+e^{-i u}\right) & 8 \sqrt{2} \\
\frac{1}{\sqrt{2}}\left(1+e^{-i u}\right)\left(-1+10 e^{-i u}-e^{-2 i u}\right) & -3+10 e^{-i u}-3 e^{-2 i u}
\end{array}\right),
$$

and which is normalized by $\left\|\phi_{1}\right\|_{L_{1}}=\sqrt{2} / 3$. These conditions define $\Phi$ uniquely, and we find $\operatorname{supp} \phi_{1}=[0,1], \operatorname{supp} \phi_{2}=[0,2]$. The space $S(\Phi)$ provides approximation order 2 . In particular, one can show, [SS], that

$$
B_{2}(x)=\frac{1}{\sqrt{2}}\left(\phi_{1}(x)+\phi_{1}(x-1)\right)+\phi_{2}(x)
$$

where $B_{2}$ is the hat function with support $[0,2]$. Choosing $F=\left(D^{2} \phi_{1}, \delta\right)^{T}$, where $\delta$ denotes the Dirac distribution, it follows that $\Phi \mapsto F$ is a good factorization process (of order 2), since from

$$
\widehat{B_{2}} \widehat{F}=\left(\begin{array}{cc}
\left(1-e^{-i \cdot}\right)^{2} \widehat{\phi}_{1} \\
\widehat{B_{2}}
\end{array}\right)=\left(\begin{array}{cc}
\left(1-e^{-i \cdot}\right)^{2} & 0 \\
\frac{1}{\sqrt{2}}\left(1+e^{-i \cdot}\right) & 1
\end{array}\right) \widehat{\Phi}
$$

it follows that $S\left(B_{2} * F\right)=S(\Phi)$, while $\widehat{F}(0)=(0,1)^{T} \neq 0$. Further, $\widehat{\Phi}(u)=(i u)^{-2} \mathbf{C}(u) \widehat{F}(u)$ with

$$
\mathbf{C}(u)=\left(\begin{array}{cc}
1 & 0 \\
-\frac{1}{\sqrt{2}}\left(1+e^{-i u}\right) & \left(1-e^{-i u}\right)^{2}
\end{array}\right) .
$$

The symbol $\mathbf{Q}$ of $F$ is then found with the aid of the relation $4 \mathbf{P ~ C}=\mathbf{C}(2 \cdot) \mathbf{Q}$ :

$$
\mathbf{Q}(u)=\frac{1}{5}\left(\begin{array}{cc}
-2\left(1+e^{-i u}\right) & 8 \sqrt{2}\left(1-e^{-i u}\right)^{2} \\
0 & 5
\end{array}\right)
$$

We can now check that the matrix $\mathbf{C}$ satisfies the conditions asserted in Theorem 3.2: indeed, $\operatorname{det} \mathbf{C}$ vanishes to order 2 at 0 , and choosing the superfunction $\psi:=B_{2}$ (which satisfies $\widehat{\psi}=\widehat{B_{2}}=$ $\tau \widehat{\Phi}$, for $\left.\tau(u)=\left(\frac{1}{\sqrt{2}}\left(1+e^{-i u}\right), 1\right)\right)$, we find that $\tau \mathbf{C}$ as well as $D(\tau \mathbf{C})$ vanish at the origin.

Obviously, the factorization process is not unique. Using, e.g., the matrix factorization of Example 4.5 of $[\mathrm{P}]$, we obtain $\mathbf{P}(u)=4^{-1} \tilde{\mathbf{C}}(2 u) \tilde{\mathbf{Q}}(u) \tilde{\mathbf{C}}(u)^{-1}$ with

$$
\tilde{\mathbf{C}}(u)=\left(\begin{array}{cc}
\sqrt{2}\left(1+e^{-i u}\right) & -2 \sqrt{2} \\
-4 e^{-i u} & 2\left(1+e^{-i u}\right)
\end{array}\right), \quad \tilde{\mathbf{Q}}(u)=\left(\begin{array}{cc}
1 & 0 \\
\frac{1}{10}\left(-1+20 e^{-i u}-e^{-2 i u}\right) & \frac{-2}{5}\left(1+e^{-i u}\right)
\end{array}\right) .
$$

The function vector $\tilde{F}=\left(\delta, \tilde{f}_{2}\right)^{T}$ corresponding to $\tilde{\mathbf{Q}}$ satisfies the relations $D^{2} \phi_{2}=-4 \delta+2 \tilde{f}_{2}+$ $2 E^{-1} \tilde{f}_{2}$ and $D^{2} \phi_{1}=\sqrt{2}\left(\delta+E^{-1} \delta-2 \tilde{f}_{2}\right)$.

These two factorizations are still closely related in the sense that the (infinite) span of $E(F)$ coincides with that of $E(\tilde{F})$, and $\operatorname{supp} D^{2} \phi_{1}=\operatorname{supp} \tilde{f}_{2}=[0,1]$.

Our use of the above theorem (in the next section) will be as follows: our specific factorization technique there exploits a transition matrix $\mathbf{C}$ of a very special structure. That structure will be then combined with the 'universal properties' that are attributed in the theorem above to all transition matrices, and the result will be an algorithm that finds the special transition matrix $\mathbf{C}$ of our process.

As stated before, we also establish a converse theorem, concerning the passage from the symbol $\mathbf{Q}$ of some refinable vector to the symbol $\mathbf{P}$ of a refinable vector that provides high approximation order. In that theorem, we will need the following lemma: 
Lemma 3.5. Let $\mathbf{C}$ be a square $n \times n$ matrix with $2 \pi$-periodic entries. Assume that $\mathbf{C}$ is smooth at the origin, that $\operatorname{det} \mathbf{C}$ has a zero at the origin of exact order $k$, and that $\operatorname{rank} \mathbf{C}(0)=n-1$. Then, after permuting the columns of $\mathbf{C}$ if necessary, $\mathbf{C}$ can be factored into

$$
\mathbf{C}=\mathbf{C}_{k}\left(\mathbf{I}+\mathbf{T}_{k}\right),
$$

such that:

(i) $\mathbf{I}$ is the identity matrix and

$$
\mathbf{T}_{k}=\left(\begin{array}{cccc}
\left(1-e^{-i \cdot}\right)^{k}-1 & 0 & \ldots & 0 \\
t_{2} & 0 & \ldots & 0 \\
t_{3} & 0 & \ldots & 0 \\
\cdot & \cdot & \cdot & \cdot \\
t_{n} & 0 & \ldots & 0
\end{array}\right),
$$

with $t_{j}, 2 \leq j \leq n$, trigonometric polynomials with spectrum in $\{0,-1, \ldots,-(k-1)\}$.

(ii) The entries of the first column of $\mathbf{C}_{k}$ are as smooth as the corresponding entries of $\mathbf{C}$, save the fact that at the origin the former may 'lose' $k$ orders of smoothness. In particular, since $\mathbf{C}$ and $\mathbf{C}_{k}$ differ at most in their first column, $\mathbf{C}_{k}$ is a trigonometric polynomial matrix, if $\mathbf{C}$ is one.

Proof: $\quad$ Since rank $\mathbf{C}(0)=n-1$, we may assume without loss that the last $n-1$ columns of that matrix are linearly independent. It suffices to prove the $k=1$ case, and then to apply induction: indeed, suppose that the factorization is possible for $j<k$. Then, the last $n-1$ columns of $\mathbf{C}_{j}$ are the same as those of $\mathbf{C}$, hence these columns are still linearly independent in $\mathbf{C}_{j}(0)$. Also, $\mathbf{C}_{j}(0)$ is still singular, since $\operatorname{det}\left(\mathbf{I}+\mathbf{T}_{j}\right)$ has a zero at the origin of exact order $j$, and $j<k$. Therefore, the case $k=1$ applies here to extract another factor of the form $\mathbf{I}+\mathbf{T}_{1}$ from $\mathbf{C}_{j}$. The induction is completed with the observation that the product of two expressions of form $\mathbf{I}+\mathbf{T}_{j}$ and $\mathbf{I}+\mathbf{T}_{1}$ is clearly an expression of the form $\mathbf{I}+\mathbf{T}_{j+1}$. So, we assume without loss that $k=1$. Since $\mathbf{C}(0)$ is singular, there exists a vector $v=\left(1, v_{2}, v_{3}, \ldots, v_{n}\right)^{T} \in \mathbb{C}^{n}$ such that $\mathbf{C}(0) v=0$. This means that there exists a smooth vector $w$ such that $\mathbf{C} v=t w$, with $t(u):=1-e^{-i u}$. (Here 'smooth' is meant in the sense of the case $k=1$ in the theorem, i.e., that $w$ is as smooth as the entries of $\mathbf{C}$, except at the origin, where it may loose one order of smoothness.) Let $\mathbf{C}_{1}$ (respectively $\mathbf{V}$ ) be the matrix obtained from $\mathbf{C}$ (respectively $\mathbf{I}$ ) when replacing the first column by $w$ (respectively $v)$. Then the above shows that $\mathbf{C V}=\mathbf{C}_{1} \operatorname{diag}(t, 1, \ldots, 1)$. The result now follows from the fact that $\operatorname{diag}(t, 1, \ldots, 1) \mathbf{V}^{-1}$ has exactly the required form $\mathbf{I}+\mathbf{T}_{1}$ (with the first column of $\mathbf{T}_{1}$ being $\left(t-1,-v_{2}, \ldots,-v_{n}\right)^{T}$.)

Theorem 3.6. Let $\mathbf{Q}$ be the symbol of an s-refinable distribution vector $F$ with $n$ entries:

$$
\widehat{F}(s u)=\mathbf{Q}(u) \widehat{F}(u) .
$$

Let $\mathbf{C}$ be an $n \times n$ matrix whose entries are $2 \pi$-periodic bounded functions that are smooth at the origin. Assume that the following conditions are satisfied for some numbers $k>0$, and $m<k-1 / 2$.

(i) $\widehat{F}$ is smooth at the origin, and grows slowly at $\infty:|\widehat{F}(u)|=O\left(|u|^{m}\right)$.

(ii) $\mathbf{Q}(0)$ has no eigenvalue of the form $s^{l}(l \in \mathbb{N}, l \geq k)$.

(iii) $\operatorname{det} \mathbf{C}$ has a $k$-fold zero at the origin, and $\operatorname{rank} \mathbf{C}(0)=n-1$.

(iv) While $\widehat{F}(0) \neq 0, \mathbf{C} \widehat{F}$ has a $k$-fold zero at the origin.

Then the matrix

$$
\mathbf{P}(u):=\frac{1}{s^{k}} \mathbf{C}(s u) \mathbf{Q}(u) \mathbf{C}(u)^{-1}
$$


is a symbol of a uniquely determined (up to multiplicative constant) s-refinable vector $\Phi \subset L_{2}$ whose corresponding $S(\Phi)$ provides approximation order $k$. Moreover, the functions in $\Phi$ decay at $\pm \infty$ at the same rates as the distributions in $F$ (in particular, $\Phi$ is compactly supported if $F$ is compactly supported) provided that $\mathbf{C}$ is a trigonometric polynomial matrix.

Proof: We note that the vector $\Phi$ is obtained from the inverse transform $\Phi_{0}$ of $\mathbf{C} \widehat{F}$ by a $k$-fold integration, i.e., $\widehat{\Phi}(u)=(i u)^{-k} \widehat{\Phi}_{0}(u)=(i u)^{-k} \mathbf{C}(u) \widehat{F}(u)$ (this is due to the appearance of the $s^{-k}$ factor in the definition of $\left.\mathbf{P}\right)$.

We first want to show that the vector $\Phi$ is uniquely determined by $\mathbf{P}$, and for that rewrite (3.7) as $\mathbf{P C}=s^{-k} \mathbf{C}(s \cdot) \mathbf{Q}$, which gives at the origin the relation

$$
\mathbf{P}(0) \mathbf{C}(0)=s^{-k} \mathbf{C}(0) \mathbf{Q}(0) .
$$

We recall that $\Phi$ can be a refinable vector with symbol $\mathbf{P}$ only if $\mathbf{P}(0)$ has an eigenvalue of the form $\mu=s^{l}, l \geq 0$ (cf. [HC], [JS]; the statements there are confined to compactly supported $\Phi$ and dyadic dilations, but the argument extends verbatim to arbitrary dilations, and to the case when $\mathbf{P}$ and $\widehat{\Phi}$ are merely smooth at the origin). Let $y$ be any left eigenvector of $\mathbf{P}(0)$ whose corresponding eigenvalue is a power $s^{l}$ of $s$. Then (3.8) implies that $s^{k+l} y \mathbf{C}(0)=y \mathbf{C}(0) \mathbf{Q}(0)$. Since $s^{k+l}$ cannot be an eigenvalue of $\mathbf{Q}(0)$ (because of assumption (ii)), we conclude that $y \mathbf{C}(0)=0$. Since rank $\mathbf{C}(0)=n-1$, it follows further that $y$ is uniquely determined (up to a multiplication by a scalar). This implies that there exists a unique right eigen pair of $\mathbf{P}(0)$ of the form $\left(s^{l}, x\right), l \geq 0$, and from that one easily concludes the uniqueness. Indeed, given a solution $\Phi$, one observes from the relation $\widehat{\Phi}(s \cdot)=\mathbf{P} \widehat{\Phi}$ that $\left(s^{j}, D^{j}(\widehat{\Phi})(0)\right)$ is a right eigen pair of $\mathbf{P}(0)$, with $j$ the first derivative of $\widehat{\Phi}$ that does not vanish at the origin. Thus, the uniqueness of the right eigenpair $\left(s^{l}, x\right)$ implies that, given two different solutions $\Phi$ and $\Psi$, they vanish both to same order $j$ at the origin, and $D^{j}(\widehat{\Phi})(0)=D^{j}(\widehat{\Psi})(0)=x$. But, then, the transform of the non-trivial solution $\Phi-\Psi$ has a zero at the origin of order $j+1$, hence $\mathbf{P}(0)$ has the eigenvalue $s^{j+1}$, which is a contradiction.

Since $\mathbf{C}$ is bounded, it follows from the slow growth assumption on $\widehat{F}$ that $\left|\widehat{\Phi}_{0}(u)\right|=O\left(|u|^{m}\right)$, hence that $|\widehat{\Phi}(u)|=O\left(|u|^{m-k}\right)$. Since $m-k<-1 / 2$, we conclude that $\Phi \subset L_{2}$.

If $\mathbf{C}$ is a trigonometric polynomial matrix, then $\Phi_{0}$ lies in the finite span of the shifts of $F$; in particular $\Phi_{0}$ decays at $\pm \infty$ (at least) at the same rate as $F$. Since condition (iv) above implies that $\widehat{\Phi}_{0}$ vanishes to order $k$ at the origin, it easily follows that $\Phi$ also decays at $\pm \infty$ at that same rate (with the usual exception: if $\Phi_{0}$ decays at $\pm \infty$ at an algebraic rate $l$, the decay rate of $\Phi$ may only be $l-k$.)

In order to prove that $S(\Phi)$ provides approximation order $k$, we invoke Lemma 3.5: since our matrix $\mathbf{C}$ here satisfies the assumptions made in the lemma, we can factor $\mathbf{C}$ into $\mathbf{C}_{k}(\mathbf{I}+\mathbf{T})$, with $\mathbf{T}$ being in the form of $\mathbf{T}_{k}$ in the lemma. Note that the first entry of a vector $(\mathbf{I}+\mathbf{T}) \widehat{F}$ is of the form $\left(1-e^{-i u}\right)^{k} \widehat{\eta}_{1}(u)$, with $\eta_{1}$ the first entry of $F$. This implies that the first entry in the inverse transform of $(\mathbf{I}+\mathbf{T}) \hat{F}$ is $\nabla^{k} \eta_{1}$. When applying the $k$-fold integration, we obtain a vector $\Phi_{1}$ whose first entry is $B_{k} * \eta_{1}$. Note that

$$
\widehat{\Phi}=\mathbf{C}_{k} \widehat{\Phi}_{1} .
$$

Now, $\mathbf{C}_{k}(0)$ is invertible, hence $\mathbf{C}_{k}$ is invertible on some neighborhood $\Omega$ of the origin. Let $S$ (respectively $S_{1}$ ) be the set of all functions in $S(\Phi)$ (respectively, $S\left(\Phi_{1}\right)$ ) whose Fourier transform is supported on $\Omega+2 \pi \mathbb{Z}$. The relations $\widehat{\Phi}=\mathbf{C}_{k} \widehat{\Phi}_{1}$, and $\widehat{\Phi}_{1}=\mathbf{C}_{k}^{-1} \widehat{\Phi}$, which are valid on $\Omega+2 \pi \mathbb{Z}$, show, [BDR2], that $S=S_{1}$. On the other hand, the general theory of approximation orders of FSI spaces, [BDR1,BDR2], implies that the approximation orders provided by any $S(F)$ are determined by the restriction of $\widehat{F}$ to $\Omega+2 \pi$ Z. Combining all the above, we conclude that the approximation orders provided by $S(\Phi)$ equal those provided by $S\left(\Phi_{1}\right)$. 
We now recall that one of the entries of $\Phi_{1}$ is of the form $\phi_{1}:=B_{k} * \eta_{1}$, and show that the subspace $S\left(\phi_{1}\right)$ of $S\left(\Phi_{1}\right)$ already provides approximation order $k$. For that we observe first that $\widehat{\eta}_{1}(0) \neq 0$ (otherwise, since $\mathbf{C}(0) \widehat{F}(0)=0$ by assumption, we get from the linear independence of the last $n-1$ columns of $\mathbf{C}(0)$ that $\widehat{F}(0)=0$.) Thus, [BDR1], in order to prove that $S\left(\phi_{1}\right)$ provides approximation order $k$, it suffices to show that $\sum_{j \in 2 \pi \mathbb{Z} \backslash 0}\left|\widehat{\phi_{1}}(u+j)\right|^{2}=O\left(|u|^{2 k}\right)$, near the origin. Using the fact that $\left|\widehat{B}_{k}(u)\right|^{2}=\sin ^{2 k}(u / 2)|u / 2|^{-2 k}$, that requirement is reduced to showing that $\sum_{j \in 2 \pi \mathbb{Z} \backslash 0}\left|\widehat{\eta}_{1}(u+j)\right|^{2}|u+j|^{-2 k}$ is bounded near the origin. However, that follows from the slow growth of $\widehat{\eta}_{1}$, together with the fact that $m-k<-1 / 2$.

Again, the conditions in the theorem can be simplified under various additional assumptions on the refinable vectors. We provide here a sample result in this direction, in which we study the following additional feature of stability.

Corollary 3.9. Assume that $F$ is a compactly supported refinable vector, with symbol $\mathbf{Q}$, whose shifts are pre-stable, and let $\mathbf{C}$ be a trigonometric polynomial matrix which is non-singular everywhere on $(0,2 \pi)$. If (i-iv) of Theorem 3.6 are valid then the solution $\Phi$ of Theorem 3.6 is compactly supported and has stable shifts, provided that the zero $\operatorname{det} \mathbf{C}$ has at the origin is exactly of order $k$.

Proof: The existence of a unique solution was proved in Theorem 3.6, and the argument that proves the compact support of $\Phi$ is sketched there, too. So, it remains to show that the shifts of $\Phi$ are stable. Since $\Phi$ has compact support, the stability requirement is equivalent to pre-stability (see Corollary 2.11 and the discussion following it), i.e., equivalent to the linear independence, for each $\theta \in[0,2 \pi)$, of the sequences

$$
c_{\theta, \phi}: 2 \pi \mathbb{Z} \mapsto \mathbb{C}: l \mapsto \widehat{\phi}(\theta+l), \quad \phi \in \Phi .
$$

For $\theta \in(0,2 \pi)$ that linear independence is clear from the relation $\widehat{\Phi}(\theta+l)=(i(\theta+l))^{-k} \mathbf{C}(\theta) \widehat{F}(\theta+l)$ (since $\mathbf{C}(\theta)$ is invertible, and the sequences $l \mapsto \widehat{f}(\theta+l), f \in F$ are linearly independent, by the pre-stability assumption on the shifts of $F$ ). It remains, thus, to show the linear independence of $\{\widehat{\phi}(l)\}_{l \in 2 \pi \mathbb{Z}}, \phi \in \Phi$.

For that, note first that the restriction to $2 \pi \mathbb{Z} \backslash 0$ of $\widehat{\Phi}$ has rank $n-1$ : indeed, assume that $y \widehat{\Phi}(l)=0$, for every $l \in 2 \pi \mathbb{Z} \backslash 0$. Then $y \mathbf{C}(0) \widehat{F}(l)=0$, for every $l \in 2 \pi \mathbb{Z}$ (with the case $l=0$ follows from the assumed fact that $\mathbf{C}(0) \widehat{F}(0)=0)$. The pre-stability assumption on $F$ then implies that $y \mathbf{C}(0)=0$; however, $\mathbf{C}(0)$ has rank $n-1$, by assumption, hence such $y$ is (essentially) unique. Consequently, in order to complete the proof here we need to show that, $y \widehat{\Phi}(0) \neq 0$, for the unique $y$ in $\operatorname{ker} \mathbf{C}(0)^{*}$.

Let $\mathbf{E}:=q \mathbf{C}^{-1}$, with $q:=\operatorname{det} \mathbf{C}$. Then the entries of $\mathbf{E}$ are trigonometric polynomials, hence $\mathbf{E C}$ as well as $\mathbf{C E}$ are continuous everywhere. Since off the origin $\mathbf{E C}=\mathbf{C E}=q \mathrm{I}$ (with $\mathrm{I}$ the identity), we conclude then that $\mathbf{E}(0) \mathbf{C}(0)=\mathbf{C}(0) \mathbf{E}(0)=\mathbf{0}$. However, rank $\mathbf{C}(0)=n-1$, with the corresponding left (right) eigenvector being $y(\widehat{F}(0)$, due to assumption (iv)), hence, necessarily $\mathbf{E}(0)=c \widehat{F}(0) y$, for some non-zero $c$. Combining these observations with the identity

$$
q(u) \mathbf{C}^{-1}(u) \widehat{\Phi}(u)=(i u)^{-k} q(u) \widehat{F}(u)
$$

(which is valid everywhere off the origin), we obtain, by taking $u \rightarrow 0$, that

$$
(c y \widehat{\Phi}(0)) \widehat{F}(0)=\left(\lim _{u \rightarrow 0}(i u)^{-k} q(u)\right) \widehat{F}(0),
$$

i.e., $y \widehat{\Phi}(0)=\left(\lim _{u \rightarrow 0}(i u)^{-k} q(u)\right) / c$. Since we assume that the order of the zero $\operatorname{det} \mathbf{C}$ has at the origin is exactly $k$, we conclude that $y \widehat{\Phi}(0) \neq 0$. 
Example: spline functions. Take $\widehat{F}(u)=\left(1, i u, \ldots,(i u)^{n-1}\right)^{T}$ and let $k \geq n$. Then obviously, $F$ is compactly supported, has pre-stable integer shifts and $F$ is $s$-refinable with symbol $\mathbf{Q}(u)=$ $\operatorname{diag}\left(1, s, s^{2}, \ldots, s^{n-1}\right)$. Let further, $\mathbf{C}=\mathbf{C}_{k}\left(\mathbf{T}_{k}+\mathbf{I}\right)$ with

$$
\mathbf{T}_{k}(u)=\left(\begin{array}{cccc}
\left(1-e^{-i u}\right)^{k}-1 & 0 & \ldots & 0 \\
t_{2}(u) & 0 & \ldots & 0 \\
t_{3}(u) & 0 & \ldots & 0 \\
\cdot & \cdot & \cdot & \cdot \\
t_{n}(u) & 0 & \ldots & 0
\end{array}\right),
$$

where each $t_{j}, 2 \leq j \leq n$, lies in the span of $\left(e^{-i l u}\right)_{l=0}^{k-1}$ and is determined by $D^{l} t_{j}(0)=$ $-\delta_{l, j-1} i^{j-1}(j-1)$ ! for $l=0, \ldots, k-1$. Then indeed, $\left.D^{l}\left[\left(\mathbf{T}_{k}(u)+\mathbf{I}\right) \widehat{F}(u)\right]\right|_{u=0}=0$ for $l=0, \ldots, k-1$, and conditions (ii)-(iv) of Theorem 3.6 are satisfied.

In the special case that $\mathbf{C}_{k}$ is a matrix of trigonometric polynomials, the vector $\Phi$ which is determined by the symbol $\mathbf{P}=s^{-k} \mathbf{C}(s \cdot) \mathbf{Q} \mathbf{C}^{-1}$ is a vector of compactly supported spline functions of degree $k-1$ with integer knots.

Letting for instance $n=2$, i.e., $\widehat{F}(u)=(1, i u)^{T}$, and $k=2, \mathbf{C}_{k}=\mathbf{I}$, we obtain $t_{2}(u)=e^{-i u}-1$ and

$$
\widehat{\Phi}(u)=\left(\begin{array}{c}
\widehat{\phi}_{1}(u) \\
\widehat{\phi}_{2}(u)
\end{array}\right)=\frac{1}{(i u)^{2}}\left(\begin{array}{cc}
\left(1-e^{-i u}\right)^{2} & 0 \\
-1+e^{-i u} & 1
\end{array}\right) \widehat{F}(u),
$$

leading to

$$
\phi_{1}(x)=\left\{\begin{array}{cc}
x & x \in(0,1] \\
2-x & x \in(1,2] \\
0 & x \notin(0,2]
\end{array}, \quad \phi_{2}(x)=\left\{\begin{array}{cc}
1-x & x \in(0,1] \\
0 & x \notin(0,1]
\end{array} .\right.\right.
$$

The corresponding symbol $\mathbf{P}_{2}$ reads

$$
\mathbf{P}_{2}(u)=\frac{1}{s^{2}}\left(\begin{array}{cc}
\left(\frac{1-e^{-i u s}}{1-e^{-i u}}\right)^{2} & 0 \\
\frac{-\left(1-e^{-i s u}\right)+s\left(1-e^{-i u}\right)}{\left(1-e^{-i u}\right)^{2}} & s
\end{array}\right) .
$$

For $n=2, k=3$, and $\mathbf{C}_{k}=\mathbf{I}$, we find $t_{2}(u)=2^{-1}\left(-3+4 e^{-i u}-e^{-2 i u}\right)$ and

$$
\widehat{\Phi}(u)=\frac{1}{(i u)^{3}}\left(\begin{array}{cc}
\left(1-e^{-i u}\right)^{3} & 0 \\
\frac{1}{2}\left(-3+4 e^{-i u}-e^{-2 i u}\right) & 1
\end{array}\right) \widehat{F}(u)
$$

with the corresponding symbol

$$
\mathbf{P}_{3}(u)=\frac{1}{s^{k}\left(1-e^{-i u}\right)^{3}}\left(\begin{array}{cc}
\left(1-e^{-i u s}\right)^{3} & 0 \\
\frac{1}{2}\left(\left(1-e^{-i u s}\right)\left(-3+e^{-i u s}\right)+s\left(1-e^{-i u}\right)\left(3-e^{-i u}\right)\right) & s
\end{array}\right) .
$$

This time, the first entry is the cardinal B-spline of order 3 with support $[0,3]$ and for the second, we find

$$
4 \phi_{2}(x)=\left\{\begin{array}{cl}
(-3 x+4) x & x \in(0,1] \\
(2-x)^{2} & x \in(1,2] . \\
0 & x \notin(0,2]
\end{array} .\right.
$$




\section{Factoring the refinement symbol}

The main result of this paper, which leads to the new factorization is as follows:

Theorem 4.1. Let $\Phi$ be an s-refinable vector of $L_{2}$-functions with symbol $\mathbf{P}$ that provides approximation order $k$. Assume further that

(i) $\Phi$ satisfies the strong $H(k)$ property at $\Xi_{s}=\{2 \pi j / s: j=0, \ldots, s-1\}$.

(ii) $D^{k} \widehat{\Phi}$ grows slowly on $O+2 \pi \mathbb{Z}$, with $O$ the neighborhood that appears in the $H(k)$ condition. Then there exist $\phi_{0} \in \Phi$ and a distribution $\eta$ such that

$$
F:=\eta \cup D^{k} \Phi^{\prime}, \quad \Phi^{\prime}:=\Phi \backslash \phi_{0}
$$

is s-refinable with a symbol $\mathbf{Q}$, and the following hold:

(a) The entries of $\mathbf{Q}$ have the same smoothness as those of $\mathbf{P}$. In particular, if $\mathbf{P}$ is a matrix-valued trigonometric polynomial, then so is $\mathbf{Q}$.

(b) If $\Phi$ satisfies the strong $H(k+1)$ property, but $S(\Phi)$ does not provide approximation order $k+1$, no non-zero distribution in the finite span of $E(F)$ is in $L_{2}(\mathbb{R})$.

(c) If the shifts of $\Phi$ are $L_{2}$-stable then the shifts of $F$ are pre-stable.

(d) $\Phi \mapsto F$ is a good factorization process of order $k$, i.e., $S\left(B_{k} * F\right)=S(\Phi)$, with $B_{k}$ the $B$-spline of order $k$ and $\widehat{F}$ is continuous at 0 with $\widehat{F}(0) \neq 0$.

Since the case when $\mathbf{P}$ is polynomial (hence $\Phi$ is compactly supported) is of primary interest here, we find it suitable to restate the main theorem for this particular case:

Corollary 4.2. Let $\Phi$ be an $s$-refinable vector of compactly supported $L_{2}$-functions with trigonometric polynomial symbol $\mathbf{P}$, and assume that $S(\Phi)$ provides approximation order $k$. Assume further that $\widehat{\Phi}$ are linearly independent over $\theta+2 \pi \mathbb{Z}$, for every $\theta \in \Xi_{s}$. Then there exist $\phi_{0} \in \Phi$ and a compactly supported distribution $\eta$ such that

$$
F:=\eta \cup D^{k} \Phi^{\prime},
$$

with $\Phi^{\prime}:=\Phi \backslash \phi_{0}$, is s-refinable with a trigonometric polynomial symbol $\mathbf{Q}$, and the following hold:

(a) No entry of $F$ is in $L_{2}(\mathbb{R})$ unless $S(\Phi)$ provides approximation order $k+1$.

(b) If the shifts of $\Phi$ are $L_{2}$-stable then the shifts of $F$ are pre-stable.

(c) $F$ is a factor of $\Phi$ in the sense that $S\left(B_{k} * F\right)=S(\Phi)$, with $B_{k}$ the $B$-spline of order $k$.

Remark: the exact meaning of the 'same smoothness'. We claim in the above theorem that the new symbol $\mathbf{Q}$ is 'as smooth as' the old symbol $\mathbf{P}$ is. The precise meaning of that statement is as follows: each entry $q$ of the new mask will be proved to be in the form $p t$, with $p$ the corresponding entry of the old mask, and $t$ a rational (entry-dependent) trigonometric polynomial; the rational polynomial $t$ has the following property: first, all its poles are at $\Xi_{s}$, and second, if $t$ has a pole of order $j$ at $\xi$, then $p$ is smooth in a neighborhood of $\xi$ and has a zero of order $\geq j$ at $\xi$ itself. One then concludes that the entries of $\mathbf{Q}$ are trigonometric polynomials (respectively, analytic functions, $C^{\infty}$-functions, continuous functions) if the entries of $\mathbf{P}$ satisfy that property. However, the property $\mathbf{P} \in C^{\rho}$ is not inherited by $\mathbf{Q}$.

We turn our attention now to the proof of Theorem 4.1. For that, several preparations are needed. We start with the following definition from [R2]:

Definition 4.3. Let $k$ be a positive integer. We say that $\Phi \subset L_{2}$ has the Property $\mathbf{H}(\mathrm{k})$ if the following condition is met: $S(\Phi)$ provides approximation order $k$ for the entire $W_{2}^{k}$ whenever there exists a nonzero function $f \in W_{2}^{k-1}$ such that, for some sequence $\left(h_{i}\right)_{i}$ that decreases to zero,

$$
\operatorname{dist}\left(f, \mathcal{D}_{h}(S(\Phi))\right)=o\left(h^{k-1}\right), \quad h=h_{1}, h_{2}, \ldots
$$


If $\Phi$ is refinable, then $\operatorname{dist}\left(f, \mathcal{D}_{h}(S(\Phi))=0\right.$, for every $f \in S(\Phi)$, and every $h=s^{-m}, m$ positive integer. Thus, if the refinable $S(\Phi)$ satisfies the Property $H(k)$ and has non-zero intersection with $W_{2}^{k-1}$, it must provide approximation order $k$. The reference [R2] establishes simple sufficient conditions for the satisfaction of the $\mathrm{H}(\mathrm{k})$ property. For example, it shows that in the univariate case every local FSI space satisfies that property, regardless of the value of $k$. However, if the generators $\Phi$ are not compactly supported, more should be assumed. For example, the following is implied by the proof of Proposition 4.2 in [R2].

Result 4.4. Let $k$ be a positive integer, $\Phi$ a finite subset of $L_{2}\left(\mathbb{R}^{d}\right)$. If $\Phi$ has the strong $H(k)$ property then it also has the Property $H(k)$.

In addition to the above, we need to following (technical) lemma:

Lemma 4.5. Let $\Phi_{0}:=\left(\psi, \phi_{2}, \ldots, \phi_{n}\right)^{T}$ be an s-refinable vector of $L_{2}$-functions with a symbol $\mathbf{P}_{0}$, which satisfies the strong $H(k)$ property at $\Xi_{s}=\left\{\frac{2 \pi j}{s}: j=0, \ldots, k-1\right\}$. Further, assume that $\psi$ satisfies the Strang-Fix conditions of order $k$ (i.e., $\widehat{\psi}$ has zero of order $k$ at each point of $2 \pi \mathbb{Z} \backslash 0$, but does not vanish at the origin.) Then each of the off-diagonal entries in the first row of $\mathbf{P}_{0}$ has a $k$-fold zero at each $\xi \in \Xi_{s}$. The diagonal element of the first row has a $k$-fold zero at each $\xi \in \Xi_{s} \backslash 0$.

Proof: $\quad$ Let $v$ be the first row of $\mathbf{P}_{0}$; then $\widehat{\psi}=v(\dot{\bar{s}}) \widehat{\Phi}_{0}(\dot{\bar{s}})$. Assume first to the contrary that some derivative $D^{j}, 0 \leq j<k$ of $v$ does not vanish at some $r \in \frac{2 \pi}{s}\{1, \ldots, s-1\}$. We may assume without loss that

$$
D^{j^{\prime}} v(r)=0, \quad j^{\prime} \in\{0, \ldots, j-1\} .
$$

Now, for every $m \in 2 \pi Z$,

$$
0=D^{j} \widehat{\psi}((r+m) s)=\left(D^{j} v\right)(r) \widehat{\Phi}_{0}(r+m),
$$

with the left-most equality due to the Strang-Fix conditions, while the right-most equality due to (4.6). Since, by our strong $H(k)$ property at $r$, the sequences $\widehat{\Phi}_{0}(r+\cdot)$ are linearly independent on $2 \pi \mathbb{Z}$, we obtain the contradiction that $D^{j} v(r)=0$.

It remains to show that the non-diagonal entries of $v$ vanish to order $k$ at 0 , too. We choose in the previous argument $r=0$, and assume that $m \in 2 \pi \mathbb{Z} \backslash 0$. With $v^{\prime}$ the off-diagonal entries of the vector $v$, and $\Phi_{0}^{\prime}:=\Phi_{0} \backslash \psi$, we observe that, for $l<k, D^{l}\left(v \widehat{\Phi}_{0}\right)(m)=D^{l}\left(v^{\prime} \widehat{\Phi}_{0}^{\prime}\right)(m)$, since $\widehat{\psi}$ has a $k$-fold zero at $m$, due to its satisfaction of the Strang-Fix conditions.

Thus, we may repeat the same argument by negation as for the case $r \neq 0$, to find $j$ such that $D^{j} v^{\prime}(0) \neq 0$, while

$$
0=D^{j} \widehat{\psi}(s m)=\left(D^{j} v^{\prime}\right)(0) \widehat{\Phi_{0}^{\prime}}(m) .
$$

In order to reach the desired contradiction, we need thus to know that the entries of $\widehat{\Phi}_{0}^{\prime}$ are linearly independent on $2 \pi Z \backslash 0$. Suppose that there is a non-trivial linear combination $\widehat{\phi}$ of span $\widehat{\Phi}_{0}^{\prime}$ that vanishes on $2 \pi \mathbb{Z} \backslash 0$. Since $\widehat{\psi}$ also vanishes there, then a non-trivial linear combination of $\widehat{\psi}$ and $\widehat{\phi}$ must vanish at all the $2 \pi$-integers. However, the strong $H(\mathrm{k})$ property that we assume here implies, inter alia, that $\widehat{\Phi}_{0}$ are linearly independent over $2 \pi \mathrm{Z}$. 


\section{Proof of Theorem 4.1.}

We first invoke Result 2.3 and replace one of the entries of $\Phi$ by the superfunction $\psi$ of that result. It is elementary to verify that the new vector still satisfies the strong $H(k)$ property. Also, Result 2.5 tells us that the symbol of the new vector is obtained by multiplying $\mathbf{P}$ by matrix-valued trigonometric polynomials, hence the new mask maintains all smoothness properties of $\mathbf{P}$. We denote the new vector by $\Phi_{0}=\psi \cup \Phi^{\prime}$ and its symbol by $\mathbf{P}_{0}$.

Next, by Theorem 2.6 there exists a tempered distribution $\eta$ such that $\psi=B_{k} * \eta$. We define

$$
F:=\left(\eta, D^{k} \Phi^{\prime}\right) \text {. }
$$

We will show that this vector $F$ is refinable with mask $\mathbf{Q}$, and that $(F, \mathbf{Q})$ satisfy the requirements (a-d). The most immediate condition is, perhaps, (d): upon convolving $F$ with $B:=B_{k}$, we obtain

$$
B * F=\left(\psi, \nabla^{k} \Phi^{\prime}\right)=: \Phi_{1} .
$$

As easily follows from Theorem 1.7 of [BDR2], $S\left(\nabla^{k} \Phi^{\prime}\right)=S\left(\Phi^{\prime}\right)$, and thus $S\left(\Phi_{1}\right)=S\left(\Phi_{0}\right)$. It is also straightforward to see that $S\left(\Phi_{0}\right)=S(\Phi)$. The continuity of $\widehat{F}$ at the origin follows from the continuity of $\widehat{\Phi}$, the latter being granted by the strong $\mathrm{H}(\mathrm{k})$ property; finally, $\widehat{F}(0) \neq 0$, since $\widehat{\eta}(0) \neq 0$ (since $\widehat{\psi}(0) \neq 0$, by Result 2.3 ). Altogether, we obtain (d).

In order to prove the refinability of $F$ (and in order to find its mask $\mathbf{Q}$ ), it is convenient to inspect first the vector $\Phi_{1}$ (see above). We note that any vector $G$ that generates $S(\Phi)$ (in the sense that $S(G)=S(\Phi)$ ) is refinable, hence so is $\Phi_{1}$. However, we would like to find its mask. For that, note that $\widehat{\Phi}_{1}=\mathbf{D} \widehat{\Phi}_{0}$ with $\mathbf{D}$ the diagonal matrix $\mathbf{D}(u):=\operatorname{diag}\left(1,\left(1-e^{-i u}\right)^{k}, \ldots,\left(1-e^{-i u}\right)^{k}\right)$, hence indeed $\Phi_{1}$ is refinable with symbol

$$
\mathbf{P}_{1}:=\mathbf{D}(s \cdot) \mathbf{P}_{0} \mathbf{D}^{-1}
$$

Now, $\Phi_{1}=B * F$, and $B$ is refinable with symbol

$$
s^{-k}\left(\frac{1-e^{-i s u}}{1-e^{-i u}}\right)^{k}
$$

Therefore, we obtain that $F$ is indeed refinable with symbol

$$
\mathbf{Q}(u)=s^{k}\left(\frac{1-e^{-i u}}{1-e^{-i s u}}\right)^{k} \mathbf{D}(s u) \mathbf{P}_{0}(u) \mathbf{D}(u)^{-1} .
$$

Introducing $\mathbf{D}_{1}(u):=\left(1-e^{-i u}\right)^{k} \mathbf{D}(u)^{-1}=\operatorname{diag}\left(\left(1-e^{-i u}\right)^{k}, 1, \ldots, 1\right)$, we can rewrite $\mathbf{Q}$ as

$$
\mathbf{Q}=s^{k} \mathbf{D}_{1}^{-1}(s \cdot) \mathbf{P}_{0} \mathbf{D}_{1} \text {. }
$$

So, only the entries in the first (i.e., $\psi$-) row of $\mathbf{P}_{0}$ are being divided when switching to $\mathbf{Q}$ : the diagonal entry is divided by $\left(\frac{1-e^{-i s u}}{1-e^{-i u}}\right)^{k}$, and the rest by $\left(1-e^{-i s u}\right)^{k}$.

We are claiming, however, that the entries of $\mathbf{Q}$ are smooth; thus we need to show that each entry in the first row of $\mathbf{P}_{0}$ vanishes to order $k$ at each of the zeros of the polynomial it is divided by. For that, we have Lemma 4.5: Since our $\psi$ satisfies the SF conditions of order $k$ (thanks to Result 2.3), and since the vector $\Phi_{1}$ satisfies the strong $H(k)$ property, we are entitled to invoke that lemma. It is elementary to check that the zeros the lemma grants us are exactly those we need in order to render the above division 'benign', and (a) is thus proved. 
Assertion (b) follows from Result 4.4 (together with the discussion preceding that result): If there exists a non-zero $L_{2}$ function $f$ in the span of $E(F)$, then $g:=B_{k} * f \in W_{2}^{k}$ and, by (d), $g$ lies in $S(\Phi)$. Therefore, the strong $\mathrm{H}(\mathrm{k}+1)$ property will imply that $S(\Phi)$ provides approximation order $k+1$.

Finally, in view of Corollary 2.11, we may assume, while proving (c), that $\widehat{\Phi}$ are linear independent over each $\theta+2 \pi \mathbb{Z}, \theta \in \mathbb{R}$ (and prove that $\widehat{F}$ has a similar property). First, in view of the definition of $\Phi_{0}$, it is clear that $\widehat{\Phi}_{0}$ is also linearly independent over each $\theta+2 \pi \mathbb{Z}$. Since

$$
\widehat{F}(u)=\frac{1}{\widehat{B}_{k}(u)} \mathbf{D}(u) \widehat{\Phi}_{0}(u)=\left(\widehat{\eta}(u),(i u)^{k} \widehat{\Phi}^{\prime}(u)\right),
$$

and $\mathbf{D}(u)$ is invertible for $u \in(0,2 \pi)$, the linear independence of $\left\{\widehat{\Phi}_{0}(u+2 \pi l)\right\} l \in \mathbb{Z}$ gives the linear independence of $\{\widehat{F}(u+2 \pi l)\} l \in \mathbb{Z}$. It remains to show that $\{\widehat{f}(2 \pi l)\}_{l \in \mathbb{Z}}(f \in F)$ are linearly independent, or equivalently, that $\{\widehat{F}(2 \pi l): l \in \mathbb{Z}\}$ has rank $n$. By the $L_{2}$-stability of $\Phi_{0}$, $\operatorname{dim}\left\{\widehat{\Phi}_{0}(2 \pi l): l \in \mathbb{Z}\right\}=n$, and, since $\widehat{\psi}(2 \pi l)=\delta_{0, l}, \operatorname{dim}\left\{\widehat{\Phi}^{\prime}(2 \pi l): l \in \mathbb{Z} \backslash\{0\}\right\}=n-1$. Thus, $\operatorname{dim}\left\{(2 \pi i l)^{k} \widehat{\Phi}^{\prime}(2 \pi l): l \in \mathbb{Z} \backslash\{0\}\right\}=n-1$ and the assertion follows since $\widehat{\eta}(0)=\widehat{\psi}(0) / \widehat{B}_{k}(0) \neq 0$.

We would like to describe an algorithm that implements the factorization whose existence is asserted in Theorem 4.1. For that purpose, we only need the following general sketch of the proof of the theorem: in order to find the factor $F$, we first replace one of the entries of $\Phi$ by the superfunction $\psi$ of Result 2.3. The new vector $\Phi_{0}$ is then related to the old one $\Phi$ via $\widehat{\Phi}_{0}=\mathbf{U}_{1} \widehat{\Phi}$, with $\mathbf{U}_{1}$ as in Result 2.5. In the next step, a $k$-fold difference is applied to each $\phi \in\left(\Phi_{0} \backslash \psi\right)$ to yield a new vector $\Phi_{1}$ which is related to $\Phi_{0}$ via

$$
\widehat{\Phi}_{1}=\mathbf{D} \widehat{\Phi}_{0}, \quad \mathbf{D}(u)=\operatorname{diag}\left(1,\left(1-e^{-i u}\right)^{k}, \ldots,\left(1-e^{-i u}\right)^{k}\right) .
$$

Finally, the B-spline $B_{k}$ is factored from each of the entries of $\Phi_{1}$ resulting in the final vector $F$. Thus

$$
\widehat{F}=\widehat{B}_{k}^{-1} \mathbf{D U}_{1} \widehat{\Phi}
$$

We state below the corollary that summarizes those observations. In that corollary we use the following (essentially known: cf. e.g. [DM], Lemma 2.1, and the case $k=1$ in Result 2.3) lemma:

Lemma 4.8. Let $\Phi$ be a refinable vector such that

(i) $\widehat{\Phi}$ is continuous on $O+2 \pi \mathbb{Z}$, with $O$ some neighborhood of the origin and $\widehat{\Phi}(0) \neq 0$;

(ii) the sequences $\widehat{\Phi}_{\mid 2 \pi \mathbb{Z}}$ are in $c_{0}(2 \pi \mathbb{Z})$ and are linearly independent.

Let $\mathbf{P}$ be the symbol of $\Phi$. Then 1 is a (geometrically) simple eigenvalue of $\mathbf{P}(0)$.

Proof: $\quad$ Since $\widehat{\Phi}(0)=\mathbf{P}(0) \widehat{\Phi}(0)$ and $\widehat{\Phi}(0) \neq 0,1$ is an eigenvalue of $\mathbf{P}(0)$. If, now, $(1, y)$ is a left-eigenpair of $\mathbf{P}(0)$, then $y \widehat{\Phi}\left(s^{m} l\right)=y \mathbf{P}(0)^{m} \widehat{\Phi}(l)=y \widehat{\Phi}(l)$ for $l \in 2 \pi \mathbb{Z} \backslash 0$ which implies (by taking $m \rightarrow \infty$, and using the decay conditions on $\widehat{\Phi}$ ) that $y \widehat{\Phi}(l)=0$. If $\left(1, y^{\prime}\right)$ is yet another eigenpair of $\mathbf{P}(0)$, then, too, $y^{\prime} \widehat{\Phi}=0$ on $2 \pi \mathbb{Z} \backslash 0$, which implies that $x \widehat{\Phi}=0$ for some non-trivial linear combination of $y, y^{\prime}$. However, $\widehat{\Phi}$ are assumed to be linearly independent on $2 \pi \mathbb{Z}$, hence $x=0$, i.e. $\left\{y, y^{\prime}\right\}$ are dependent, hence 1 is a simple eigenvalue. 
Corollary 4.9. Let $\Phi$ be an s-refinable vector of $L_{2}$-functions with symbol $\mathbf{P}$ which satisfies conditions (i), (ii) of Theorem 4.1. Suppose that $\Phi$ is ordered such that the first entry of the left (i.e., row) 1-eigenvector of $\mathbf{P}(0)$ is not zero. Assume that $S(\Phi)$ provides approximation order $k$. Then $\mathbf{P}$ admits a factorization

$$
\mathbf{P}(u)=\left(\frac{1-e^{-i s u}}{s\left(1-e^{-i u}\right)}\right)^{k} \mathbf{W}(s u)^{-1} \mathbf{Q}(u) \mathbf{W}(u)
$$

with $\mathbf{W}$ a matrix of the form

$$
\mathbf{W}(u)=\left(\begin{array}{cccc}
1 & \tau_{\phi_{2}}(u) & \ldots & \tau_{\phi_{n}}(u) \\
0 & \left(1-e^{-i u}\right)^{k} & \ldots & 0 \\
\vdots & \ddots & \ddots & \vdots \\
0 & \ldots & 0 & \left(1-e^{-i u}\right)^{k}
\end{array}\right)
$$

where $\tau_{\phi_{j}}(u), j=2, \ldots, n$, are suitable trigonometric polynomials in the span of $\left(e^{-i u j}\right)_{j=0}^{k-1}$. Moreover, the entries of $\mathbf{Q}$ have the same smoothness as those of $\mathbf{P}$ and, in particular, $\mathbf{Q}$ only has trigonometric polynomials as entries if $\mathbf{P}$ does. Finally, the factorization is a good factorization of order $k$.

Proof: The result follows directly from the discussion preceding this theorem (with $\mathbf{W}$ here being $\mathbf{D} \mathbf{U}_{1}$ ). The only part that requires verification is the statement concerning the correct ordering of the elements in $\Phi$, i.e., that we may replace the first entry of $\Phi$ by the superfunction $\psi$ of Result 2.3. For that, we recall from the proof of Theorem 4.12 in [BDR4] that $\phi \in \Phi$ is replaceable by the superfunction if, with $\sigma$ the canonical $k$-vector, the $\phi$-entry of $\sigma(0)$ is non-zero (see Result 2.2). Since, by Result 2.2(iii), $(1, \sigma(0))$ is a left (row) eigen pair of $\mathbf{P}(0)$, if follows from the simplicity of the eigenvalue 1 of $\mathbf{P}(0)$ (and from the assumption we make) that the first entry of $\sigma(0)$ does not vanish, hence that we may replace that entry by the superfunction.

Discussion: An algorithm for computing the symbol $Q$ of $F$. As said before, the practical input/output of a factorization process are the symbols $\mathbf{P}$ and $\mathbf{Q}$ (rather than the vectors $\Phi$ and $F$ ). In order to be able to compute $\mathbf{Q}$ from $\mathbf{P}$, we only need to know the transition matrix $\mathbf{C}$ (in the relation $\left.\widehat{\Phi}=(i \cdot)^{-k} \mathbf{C} \widehat{F}\right)$, since $\mathbf{Q}$ is related to $\mathbf{P}$ as in (3.7). Comparing (3.7) with Corollary 4.9 , we find that $\mathbf{C}^{-1}(u)=\left(1-e^{-i u}\right)^{-k} \mathbf{W}(u)$, and one can then check directly that

$$
\mathbf{C}=\left(\begin{array}{ccccc}
\left(1-e^{-i \cdot)^{k}}\right. & -\tau_{2} & & \ldots & -\tau_{n} \\
0 & 1 & 0 & \ldots & 0 \\
\vdots & 0 & \ddots & \ddots & \vdots \\
\vdots & & \ddots & \ddots & 0 \\
0 & \ldots & & 0 & 1
\end{array}\right)
$$

with $\tau_{j}, j=2, \ldots, n$, trigonometric polynomials with spectrum in $\{0,-1, \ldots,-(k-1)\}$. In order to determine the trigonometric polynomials $\tau=\left(\tau_{2}, \ldots, \tau_{n}\right)$, we appeal to the general conditions on a factorization matrix $\mathbf{C}$ from Theorem 3.2; specifically we invoke (c) there. As the superfunction in that result we choose the canonical one (as discussed at the beginning of section 2). We will have then (a) to find the canonical vector $\sigma$ of order $k$, and (b) to use the fact that $\sigma \mathbf{C}$ has a $k$-fold zero at the origin in order to compute the above $\tau$-polynomials. 
The second task here is straightforward: with $\sigma_{j}:=\sigma_{\phi_{j}}$, the condition about $\sigma \mathbf{C}$ requires that each $\sigma_{j}-\tau_{j} \sigma_{1}$ has a zero of order $k$ at the origin $(j=2, \ldots, n)$. Fixing $j$, and differentiating this function $m$ times $(m=0, \ldots, k-1)$ at the origin leads to a lower triangular $k \times k$ linear system $A x=b$, with $x(r)=D^{r} \tau_{j}(0), b(m)=D^{m} \sigma_{j}(0)$, and $A(m, r)=\left(\begin{array}{c}m \\ r\end{array}\right) D^{m-r} \sigma_{1}(0)(r=0, \ldots, k-1$, $m \geq r)$. Note that the system is invertible since $\sigma_{1}(0) \neq 0$. One then easily finds $\tau_{j}$ from its $k$ th order Taylor expansion at the origin.

The argument above shows that we do not need to find the canonical vector $\sigma$, but only its derivatives (up to order $k-1$ ) at the origin. For that we invoke now part (ii) of Result 2.2.

As discussed before, the strong $H(k)$ property implies that 1 is a simple eigenvalue of $\mathbf{P}(0)$. Since $\sigma(0)$ is the corresponding left eigenvector, and $\widehat{\Phi}(0)$ is the corresponding right eigenvector, it follows that $\sigma(0)$ is already determined by conditions $\sigma(0) \mathbf{P}(0)=\sigma(0)$ and $\sigma(0) \widehat{\Phi}(0)=1$.

An $m$-fold differentiation of the expression $\sigma(s u) \mathbf{P}(u)-\delta_{0 l} \sigma(u)$, followed by an evaluation at $u=0$ leads to the system

$$
D^{m} \sigma(0)\left(s^{m} \mathbf{P}(0)-I\right)=-\sum_{\mu=1}^{m}\left(\begin{array}{l}
m \\
\mu
\end{array}\right) s^{m-\mu} D^{m-\mu} \sigma(0) D^{\mu} \mathbf{P}(0),
$$

and evaluation at $u=\xi, \xi \in \Xi \backslash\{0\}$, to the systems

$$
D^{m} \sigma(0)\left(s^{m} \mathbf{P}(\xi)\right)=-\sum_{\mu=1}^{m}\left(\begin{array}{l}
m \\
\mu
\end{array}\right) s^{m-\mu} D^{m-\mu} \sigma(0) D^{\mu} \mathbf{P}(\xi)
$$

The right hand side in each of the above systems requires the vectors $D^{r} \sigma(0), r=0, \ldots, m-1$, hence we can compute $D^{m} \sigma(0)$ recursively for $m=1,2, \ldots, k-1$, provided that the matrices

$$
\left[I-s^{m} \mathbf{P}(0), \mathbf{P}\left(\frac{2 \pi}{s}\right), \ldots, \mathbf{P}\left(\frac{2 \pi(s-1)}{s}\right)\right]
$$

(where $m>0$ ) do not have a common left-eigenpair $(0, w)$. Thus, it remains only to show that such an eigenpair $(0, w)$ cannot exist: the refinability of $\widehat{\Phi}$ implies that, for each $l \in 2 \pi Z \backslash \backslash$, $\widehat{\Phi}(l)=\mathbf{P}(0)^{r_{l}} \mathbf{P}(\xi) v$, for suitable integer $r_{l}, \xi \in \Xi_{s} \backslash 0$, and a vector $v \in \mathbb{C}^{n}$; hence, if an eigenpair $(0, w)$ exists, it follows that $w \perp \widehat{\Phi}(l)$. Furthermore, since $\mathbf{P}(0) \widehat{\Phi}(0)=\widehat{\Phi}(0)$, it also follows that $w \perp \widehat{\Phi}(0)$. This implies that $\widehat{\Phi}$ are linearly dependent on $2 \pi \mathbb{Z}$, hence that the Gramian matrix of $\Phi$ is singular at the origin, contradicting thereby the strong $H(k)$ property that we assume throughout.

Example: GHM-scaling functions. Consider again the GHM-scaling vector $\Phi=\left(\phi_{1}, \phi_{2}\right)^{T}$ with symbol $\mathbf{P}$ as in (3.4) for $s=2$. Then, with $y:=\left(1, \frac{\sqrt{2}}{2}\right),(1, y)$ is a left eigenpair of $\mathbf{P}(0)$ hence either entry of $\Phi$ is replaceable by the special superfunction $\psi$. Let us first compute the canonical superfunction $\psi_{0}=\sigma \Phi$, or more precisely, the canonical $\Phi$-vector $\sigma$ of order 2 . As stated in Result $2.2(\mathrm{i}, \mathrm{ii}),(\sigma(0), D \sigma(0))$ is determined by $\sigma(0) \widehat{\Phi}(0)=1$ and

$$
\begin{aligned}
\sigma(0) \mathbf{P}(0) & =\sigma(0), & \sigma(0) \mathbf{P}(\pi)=0, \\
2 D \sigma(0) \mathbf{P}(0)+\sigma(0) D \mathbf{P}(0) & =D \sigma(0) & 2 D \sigma(0) \mathbf{P}(\pi)+\sigma(0) D \mathbf{P}(\pi)=0 ;
\end{aligned}
$$


also, $\sigma(u)=(\sigma(0)-i D \sigma(0))+i D \sigma(0) e^{-i u}$. These equalities provide with $\widehat{\Phi}(0)=\frac{1}{3}(\sqrt{2}, 1)^{T}$ the vectors $\sigma(0)=(\sqrt{2}, 1)$ and $D \sigma(0)=\frac{i}{2}(\sqrt{2}, 2)$ and hence $\sigma(u)=\left(\frac{\sqrt{2}}{2}\left(3-e^{-i u}\right),\left(2-e^{-i u}\right)\right)$. If we want to replace $\phi_{1}$ by its corresponding superfunction $\psi=\psi_{1}$ (from Result 2.3), we need to compute $\tau^{\prime}=\tau_{\phi_{2}}$. The above relations lead to

$$
\begin{aligned}
\tau_{\phi_{2}}(0) & =\frac{1}{\sigma_{\phi_{1}}(0)} \sigma_{\phi_{2}}(0)=\frac{\sqrt{2}}{2} \\
D \tau_{\phi_{2}}(0) & =\frac{1}{\sigma_{\phi_{1}}(0)}\left(D \sigma_{\phi_{2}}(0)-D \sigma_{\phi_{1}}(0) \tau_{\phi_{2}}(0)\right)=\frac{i \sqrt{2}}{4}
\end{aligned}
$$

Hence, $\tau_{\phi_{2}}=\frac{\sqrt{2}}{4}\left(3-e^{-i u}\right)$. The special superfunction (of Result 2.3) corresponding to $\phi_{1}$ is then $\psi_{1}=\phi_{1}+\frac{\sqrt{2}}{4}\left(3 \phi_{2}-\phi_{2}(\cdot-1)\right)$.

The special superfunction $\psi=\psi_{2}$ corresponding to $\phi_{2}$ is obtained by computing $\tau_{\phi_{1}}$. We find, analogously as before, $\tau_{\phi_{1}}(0)=\sqrt{2}$ and $D \tau_{\phi_{1}}(0)=-i \frac{\sqrt{2}}{2}$, and hence $\tau_{\phi_{1}}(u)=\frac{\sqrt{2}}{2}\left(1+e^{-i u}\right)$. Thus, the superfunction corresponding to $\phi_{2}, \psi_{2}=\phi_{2}+\frac{\sqrt{2}}{2}\left(\phi_{1}+\phi_{1}(\cdot-1)\right)$ is the hat function $B_{2}$ (cf. Example in $\S 3$ ).

Note that according to Theorem $2.6 \psi_{1}=\psi_{2} * \eta$, with a suitable distribution $\eta$.

\section{An application: the smoothness of univariate refinable vectors}

As mentioned before, the new factorization technique leads to numerical methods for the computation of the smoothness exponent of each entry in a univariate refinable vector $\Phi$. This application is the topic of the current section. We focus on the $L_{2}$-smoothness parameter (aliased as 'Sobolev regularity'), which is defined, for a tempered distribution $\phi$, as

$$
\alpha(\phi):=\sup \left\{\alpha \in \mathbb{R}: \phi \in W_{2}^{\alpha}\right\},
$$

with $W_{2}^{\alpha}$ the usual Sobolev space. Note that we do not exclude the possibility of a negative $\alpha$.

Let $\Phi \subset L_{2}(\mathbb{R})$ be an $s$-refinable vector with mask symbol P:

$$
\widehat{\Phi}(s \cdot)=\mathbf{P} \widehat{\Phi} .
$$

A major challenge in wavelet theory is to determine the smoothness of the functions in $\Phi$ using mostly information about the mask $\mathbf{P}$. It is beyond the scope of this paper to review to any extent the enormous work that was done on this problem. We will incorporate, however, some of the most recent results on the matter from [RS], hence need to briefly review those particular results. We mention that the results of that reference apply to refinable vector functions in several variables, however, aiming at combining those techniques with our univariate factorization results, we describe the results of [RS] in a univariate context only.

The functions/distributions we study here are the components of a refinable vector $F$, particularly that of Theorem 4.1. However, this study is only the means for finding the smoothness of the entries of the original refinable $\Phi$. We rely here on the facts that almost all the entries of $\Phi$ coincide with those of $B_{k} * F$, and that 
Proposition 5.1. Let $F$ be a refinable vector of compactly supported tempered distributions with bounded symbol $\mathbf{P}$. Then, for each $f \in F$, and each $B$-spline of order $k, \alpha\left(B_{k} * f\right)=\alpha(f)+k$.

We prove this result at the end of this section (since the only proof we know involves the transfer operator, and is, surprisingly, very technical and elaborate).

The reference [RS] suggests two equivalent techniques for determining the smoothness of a refinable $\Phi$, the transfer operator approach, and the subdivision operator approach. We choose here to describe the problem in terms of conceptually simpler subdivision approach. (We stress that the subdivision operator approach is not necessarily the right approach from the computational point of view.) In order to simplify the presentation, we assume throughout the remainder of this section that the refinable vector $\Phi$ is compactly supported (but not necessarily that the mask symbol is polynomial), choose an interval $[0, a]$ that contains supp $\Phi$, and set

$$
H:=\operatorname{span}\left\{u \mapsto e^{-i r u}: r \in([-a, a] \cap \mathbb{Z})\right\} .
$$

Definition 5.2. Let $\Phi$ be $s$-refinable with mask symbol $\mathbf{P}$. The subdivision operator is a map $T^{*}$ from $H^{\Phi}$ into itself defined by

$$
T^{*}: g \mapsto \sqrt{s} P \mathbf{P}^{*} \mathcal{D}^{-1} g,
$$

with $\mathcal{D}^{-1}: g \mapsto g(s \cdot)$, and with $P$ the orthogonal projection onto $H^{\Phi}$ (say, from $\left.L_{2}(\mathrm{TT})^{\Phi}\right)$ ).

The techniques that use either the transfer operator iterations and/or the subdivision operator iterations are intrinsically numerically unstable: they attempt to compute eigenvalues that are smaller in magnitude than the spectral radius of the operator. In rough terms, there might be three different sources for this unfortunate phenomenon (i.e., that we are bound to chase a nondominant eigenvalue):

(i) The refinable $\Phi$ consists of functions, and not merely distributions. In this case, the spectrum of the subdivision operator contains large eigenvalues that are connected to approximation orders / polynomial reproduction (some of them are guaranteed to be larger than the eigenvalue we are after). Factorization solves this problem. The problem can be solved without factorization (see below) but at the cost of the above-mentioned numerical instability.

(ii) The shifts of $\Phi$ are not stable/pre-stable. This grants the subdivision operator additional irrelevant eigenvalues (that may or may not be large), hence should be suppressed, too, if large. In fact, [RS] is the first article to tackle the regularity problem without the assumption of stability. We note that in one variable a factorization method (of a completely different nature compared to the one here or that in [P]) can still be used to overcome that particular problem (cf. [R3] for a discussion of that other factorization technique in the univariate scalar case).

(iii) The attempt to find separate regularity estimates for each of the entries in $\Phi$. At the time this article is written, we know of no method (even in one dimension) for avoiding this problem.

When reading the two results below, it is useful to keep in mind the following picture: the use of the Gramian in these results is the way one suppresses (at least in theory) the eigenvalues that arise from instability (cf. (ii) above). The use of the trigonometric polynomial $u$ below is the way one suppresses the eigenvalues that arise from the positive smoothness of the entries of $\Phi$ (cf. (i) above). Finally, componentwise estimates of the smoothness are obtained by choosing different initial seeds.

We quote now two different results from [RS]: the first concerns the smoothness of $L_{2}$-refinable $\Phi$, and the second concerns the smoothness of $\Phi$ whose entries are not in $L_{2}$. We recall the notion of the Gramian as defined in Definition 1.4, and the notions of stability and pre-stability of the shifts of $\Phi$ (cf. $\S 2)$.

In what follows the Gramian $\mathbf{G}$ is considered pointwise as a quadratic form, i.e., given $v: \mathrm{T} \rightarrow$ $\mathbb{C}^{\Phi}$, the notation $\mathbf{G}(v)$ stands for the (scalar) function $t \mapsto v^{*}(t) \mathbf{G}(t) v(t)$. 
Result 5.3. Let $\Phi \subset L_{2}$ be s-refinable with mask $\mathbf{P}$ and Gramian $\mathbf{G}$. For $\phi \in \Phi$, let $1_{\phi} \in \mathbb{R}^{\Phi}$ be the vector whose $\phi$-entry is 1 and the other entries are zero. Finally, let $u(t):=\left(1-e^{-i t}\right)^{2 k}$, with $k$ any positive integer that exceeds the regularity parameter of $\Phi$. Then:

(I) Define $a(k, \phi):=\left\|\mathbf{G}\left(u T^{* k}\left(1_{\phi}\right)\right)\right\|_{L_{1}(\mathrm{~T})}^{1 / k}$, and find $a(\phi):=\limsup _{k \rightarrow \infty} a(k, \phi)$. Then the regularity parameter $\alpha(\phi)$ of $\phi$ is $\alpha(\phi)=-\frac{\log _{s} a(\phi)}{2}$.

(II) Define $a_{I}(k, \phi):=\left\|u T^{* k}\left(1_{\phi}\right)\right\|_{L_{2}(\mathrm{~T})}^{1 / k}$, and find $a_{I}(\phi):=\limsup _{k \rightarrow \infty} a_{I}(k, \phi)$. Then the regularity parameter $\alpha(\phi)$ of $\phi$ satisfies $\alpha(\phi) \geq-\log _{s} a_{I}(\phi)$, and equality holds if the shifts of $\Phi$ are $L_{2}$-stable.

Result 5.4. Let $\Phi$ be an s-refinable vector of distributions. Let $\nu$ be a "sufficiently smooth" compactly supported function, for which $\nu * \Phi \subset L_{2}$, and let $\mathbf{G}$ be the Gramian of $\nu * \Phi$.

(I) Set $b(k, \phi):=\left\|\mathbf{G}\left(T^{* k}\left(1_{\phi}\right)\right)\right\|_{L_{1}(\mathrm{TT})}^{1 / k}$, and let $b(\phi):=\limsup _{k \rightarrow \infty} b(k, \phi)$. Then the (negative) regularity parameter of $\phi$ is $-\frac{\log _{s} b(\phi)}{2}$.

(II) Set $b_{I}(k, \phi):=\left\|T^{* k}\left(1_{\phi}\right)\right\|_{L_{2}(\mathrm{~T})}^{1 / k}$, and let $b_{I}(\phi):=\limsup _{k \rightarrow \infty} b_{I}(k, \phi)$. Then the regularity parameter $\alpha(\phi)$ of $\phi$ satisfies $\alpha(\phi) \geq-\log _{s} b_{I}(\phi)$, and equality holds if the shifts of $\Phi$ are pre-stable, provided $\phi \in L_{2}$.

We want to apply the Result 5.4 for estimating the regularity parameter of the entries $\phi$ of $\Phi$ separately. Invoking our results of $\S 4$, the procedure is as follows: For a refinable function vector $\Phi$ with given mask symbol $\mathbf{P}$ and approximation order $k$, we first choose an appropriate good factorization process $\Phi \mapsto F$ of order $k$ and then compute the (negative) regularity parameter of the entries $D^{k} \phi, \phi \in \Phi$ of $F$ (recall that all the functions in the vector $F$, with the exception of one, are of this form).

Example: GHM-scaling functions. Let us compute the regularity parameter of the first entry $\phi_{1}$ of GHM-scaling function $\Phi=\left(\phi_{1}, \phi_{2}\right)^{T}$. From the example in $\S 3$, we recall that $\Phi \mapsto F$ with $F=\left(D^{2} \phi_{1}, \delta\right)$ (with $\delta$ the Dirac distribution) is a good factorization process and $F$ is refinable with

$$
\mathbf{Q}(u)=\frac{1}{5}\left(\begin{array}{cc}
-2\left(1+e^{-i u}\right) & 8 \sqrt{2}\left(1-e^{-i u}\right)^{2} \\
0 & 5
\end{array}\right) .
$$

Numerical computation of $\alpha_{k}\left(\phi_{1}\right):=-\log _{2} b_{I}\left(k, \phi_{1}\right)+2$ with

$$
b_{I}\left(k, \phi_{1}\right)=\left\|2^{k / 2} \mathbf{Q}^{*} \mathbf{Q}^{*}(2 \cdot) \ldots \mathbf{Q}\left(2^{k-1} \cdot\right)\left(1_{\phi_{1}}\right)\right\|_{L_{2}(\mathrm{~T})}^{1 / k}
$$

then gives e.g. $\alpha_{30}\left(\phi_{1}\right)=1.404585, \alpha_{50}\left(\phi_{1}\right)=1.442751, \alpha_{100}\left(\phi_{1}\right)=1.471375, \alpha_{200}\left(\phi_{1}\right)=1.485687$, $\alpha_{500}\left(\phi_{1}\right)=1.494275, \alpha_{1000}\left(\phi_{1}\right)=1.497138$.

The computation suggests that indeed the regularity parameter $\alpha\left(\phi_{1}\right)$ is not greater then 1.5. On the other hand, a computation of the regularity parameter of $\phi_{1}, \phi_{2}$ together (with the approach by transfer operator, see e.g. [J], Example 4.2) gives the Sobolev regularity parameter 1.5.

Proof of Proposition 5.1. The fact that $\alpha\left(B_{k} * f\right) \geq \alpha(f)+k$ is trivial: $D^{k}\left(B_{k} * f\right)=\nabla^{k} f$, and obviously $\alpha\left(\nabla^{k} f\right) \geq \alpha(f)$. The refinability assumption plays no role here.

For the converse we let $T$ be the transfer operator defined as follows: let $F$ be refinable with mask $\mathbf{Q}$. Then $T$ is defined on all $F \times F$ matrices $\mathbf{H}$ whose entries are in $L_{2}$ (TT) as

$$
T \mathbf{H}:=\sum_{m=0}^{s-1}\left(\mathbf{Q H Q}^{*}\right)\left(\frac{\cdot+2 \pi m}{s}\right) .
$$


Section 3 of [RS] proves that if $\nu$ is a sufficiently smooth compactly supported function with meanvalue 1 , and if $u$ is a $2 \pi$-periodic function which vanishes at the origin to a 'high enough' order, and is positive in some punctured neighborhood of the origin, then, with $\mathbf{G}_{\nu}$ the Gramian of $\nu * F$, and with $g_{\phi, m}$ the $\phi$-diagonal entry of $T^{m}\left(u \mathbf{G}_{\nu}\right)$, we have that

$$
\alpha(\phi)=-\frac{\log _{s} \limsup \sup _{m \rightarrow \infty}\left\|g_{\phi, m}\right\|_{L_{1}(\mathrm{TT})}^{1 / m}}{2} .
$$

With loss, we assume that $\nu$ is of the form $\nu=B_{k} * \nu$ ', with $\nu$ ' some other 'sufficiently smooth' mollifier.

Now, suppose that we replace $F$ by $\Phi:=B_{k} * F$, and set out to find the smoothness of the entries of $\Phi$ using the above recipe. Since of $\nu^{\prime} * \Phi=\nu * F$, we may still use the same Gramian $G_{\nu}$ as before. As the other mollifier $u^{\prime}$ we choose $u^{\prime}=\sin (\cdot / 2)^{2 k} u$, with $u$ the function used in the $F$-process. Let $\mathbf{P}$ be the symbol of $\Phi$, then

$$
\mathbf{P H P}^{*}=\left(\frac{\sin (s \cdot / 2)}{s \sin (\cdot / 2)}\right)^{2 k} \mathbf{Q H Q}^{*} \text {. }
$$

This implies, with $T_{1}$ the transfer operator associated with $\Phi$, that

$$
T_{1}\left(u^{\prime} \mathbf{G}_{\nu}\right)=s^{-2 k} \sin ^{2 k}(\cdot / 2) T\left(u \mathbf{G}_{\nu}\right),
$$

hence that

$$
T_{1}^{m}\left(u^{\prime} \mathbf{G}_{\nu}\right)=s^{-2 k m} \sin ^{2 k}(\cdot / 2) T^{m}\left(u \mathbf{G}_{\nu}\right),
$$

Finally, since $F$ and $\Phi$ are compactly supported, [RS] shows that the matrices $T_{1}^{m}\left(u^{\prime} \mathbf{G}_{\nu}\right)$ and $T^{m}\left(u \mathbf{G}_{\nu}\right), m=1,2, \ldots$ all lie in some finite dimensional space. Since all norms are equivalent on finite dimensional spaces,

$$
\limsup _{m \rightarrow \infty}\left\|g_{\phi, m}\right\|_{L_{1}(\mathrm{TT})}^{1 / m}=\limsup _{m \rightarrow \infty}\left(\int_{\mathrm{T}} \sin ^{2 k}(\cdot / 2) g_{\phi, m}\right)^{1 / m} .
$$

The result now follows.

\section{References}

[BDR1] de Boor, C., R. A. DeVore, and A. Ron, Approximation from shift-invariant subspaces of $L_{2}\left(\mathbb{R}^{d}\right)$, Trans. Amer. Math. Soc. 341 (1994), 787-806.

[BDR2] de Boor, C., R. A. DeVore, and A. Ron, The structure of finitely generated shift-invariant spaces in $L_{2}\left(\mathbb{R}^{d}\right)$, J. Funct. Anal. 119 (1994), 37-78.

[BDR4] de Boor, C., R. A. DeVore, and A. Ron, Approximation orders of FSI spaces in $L_{2}\left(\mathbb{R}^{d}\right)$, Constr. Approx., to appear.

[CDP] Cohen, A., I. Daubechies, and G. Plonka, Regularity of refinable function vectors, J. Fourier Anal. Anal. 3 (1997), 295-324.

[DM] Dahmen, W., and C. A. Micchelli, Biorthogonal wavelet expansions, Constr. Approx. 13 (1997), 293-328.

[GHM] Geronimo, J. S., D. P. Hardin, and P. R. Massopust, Fractal functions and wavelet expansions based on several scaling functions, J. Approx. Theory 78 (1994), 373-401.

[HC] Heil, C., and D. Colella, Matrix refinement equations: Existence and uniqueness, J. Fourier Anal. Anal. 2 (1996), 363-377. 
[JM] Jia, R. Q, and C. A. Micchelli, Using the refinement equation for the construction of prewavelets II: powers of two, Curves and Surfaces P. J. Laurent, A. Le Méhauté, and L. L. Schumaker eds., Academic Press, New York, 1991, 209-246.

[J] Jiang, Q., On the regularity of matrix refinable functions, SIAM J. Math. Anal., to appear.

[JS] Jiang, Q., and Z. Shen, On existence and weak stability of matrix refinable functions, Constr. Approx., to appear.

[P] Plonka, G., Approximation order provided by refinable function vectors, Constr. Approx. 13 (1997), 221-244.

[R1] Ron, A., Factorization theorems for univariate splines on regular grids, Israel Journal of Mathematics 70 (1990), 48-68.

[R2] Ron, A., Smooth refinable functions provide good approximation orders, SIAM J. Math. Anal. 28 (1997), 731-748.

[R3] Ron, A., Characterizations of linear independence and stability of the shifts of a univariate refinable function in terms of its refinement mask, CMS Tech. Rep. 93-3, University of Wisconsin-Madison, Sept. 92.

[RS] Ron, A., and Z. Shen, The sobolev regularity of refinable functions, preprint 1997.

[SF] Strang, G., and G. Fix, A Fourier analysis of the finite element variational method, Constructive Aspects of Functional Analysis (G. Geymonat, ed) C.I.M.E. II Ciclo, 1973, 793-840.

[SS] Strang, G., and V. Strela, Short wavelets and matrix dilation equations, IEEE Trans Acoustic., Speech, and Signal Processing 43 (1995), 108-115. 